\title{
Static Electric Fields in an Infinite Plane Condensor with One or Three Homogeneous Layers
}

\author{
T. Heubrandtner ${ }^{1)}$, B. Schnizer ${ }^{2)}$, C. Lippmann ${ }^{3)}$, W. Riegler ${ }^{3)}$
}

\begin{abstract}
Various expressions are derived for the Green's functions for a point charge in an infinite plane condensor comprising one or three homogeneous isolating parallel dielectric layers. In view of numerical evaluations needed for calculating space charge effects in detectors (e.g. RPC's) the merits of these (series and integral) representations are discussed. It turns out that in most cases the integral representations are more favourable after their convergence has been improved. This is done by subtracting simple terms having the same asymptotic behaviour as certain too slowly converging terms and adding closed expressions resulting from the integration of the simple terms. The method is demonstrated in some detail. In addition analytic expressions for the weighting field of a strip electrode are derived which allow calculation of induced signals and crosstalk.
\end{abstract}

1) Institut für Theoretische Physik, TU Graz, Graz, Austria, supported by Fonds zur Förderung der wissenschaftlichen Forschung, Vienna, Austria, Grant Nr. P10648-NAW

2) Institut für Theoretische Physik, TU Graz, Graz, Austria

3) EP Division, CERN, CH-1211 Geneva 23, Switzerland 


\section{Introduction}

The electric field solutions of a point charge in an infinite plane condensor are necessary to calculate space charge effects in various kinds of particle detectors. Fig.1 shows the two geometries described in this report. The point charge is at position $x^{\prime}, y^{\prime}, z^{\prime}$. The signal induced on a strip electrode (Fig. 2) by the movement of a charge in the condensor can be calculated by a so called weighting field, i.e. the electric field in the condensor if the electrode is put to $1 \mathrm{~V}$ while all the other electrodes are grounded. These solutions are also given in this report.

The capacitor with one homogeneous layer resembles e.g. the geometry of a noble liquid calorimeter cell [1] or Parallel Plate Chamber [2] while the structure with three homogeneous layers resembles the geometry of a Resistive Plate Chamber [3].

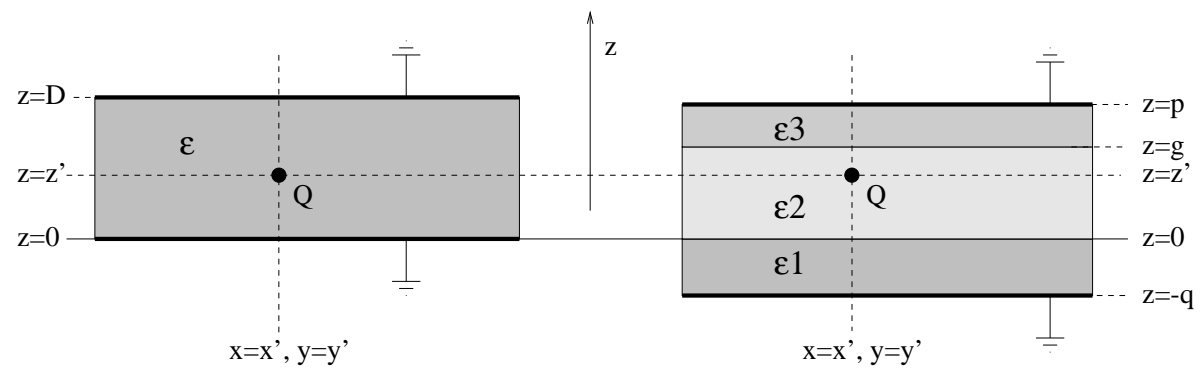

Figure 1: The two geometries discussed in this report. The point charge $Q$ is at position $x^{\prime}, y^{\prime}, z^{\prime}$.

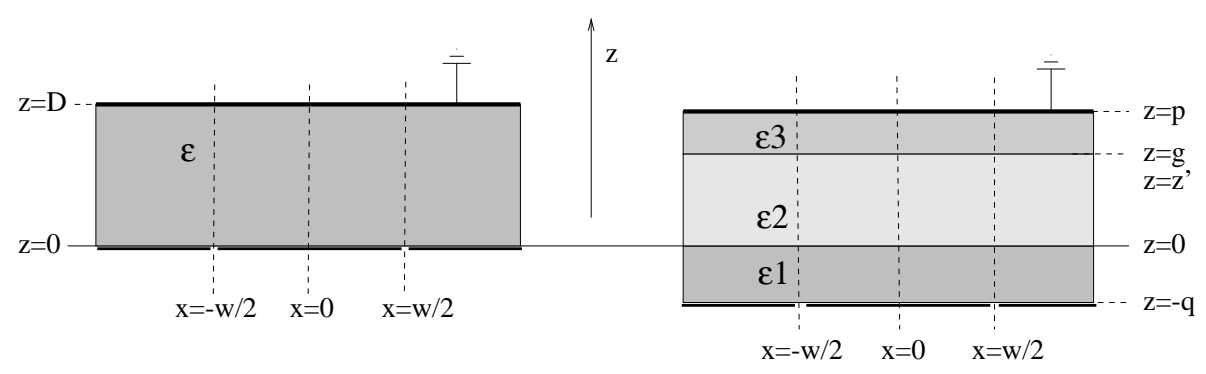

Figure 2: Readout strip geometries discussed in this report. The strips are infinitely long in $y$ and the gap between the strips is assumed to be 0 .

In this report we describe several representations of the field solutions for these geometries that have different convergence properties. The series expansions of the solutions show good convergence properties far from the vertical axis (parallel to $z$-axis) passing through the charge but very slow convergence close to this axis. The integral representations converge poorly in a horizontal plane containing the charge. This defect can be cured by subtracting simple terms having the same asymptotic behaviour as the slowly convergent terms from the integrand and compensating this by adding terms obtained from integrals over the subtrahends. These new integral representations converge very well for all points.

The one-layer condensor is treated first in order to illustrate the behaviour of the different representations and the method of curing the deficiencies (sect. 2). Thereafter the integral representations for the three-layer condensor are derived in sect. 3. In both cases expressions are given for the weighting field due to a single infinitely thin and infinitely long strip. A further approximation is that the gaps separating this strip from its neighbours are neglected, so the strip is just a sub-domain of a continuous metallic sheet. 
The analytic calculations needed for the three-layer condensor are quite involved and have been done with Mathematica [4]. But all the resulting formulae are given completely.

\section{Potential of a Point Charge in a Condensor Filled by a Homogeneous Dielectric}

A plane condensor filled with a homogeneous, constant, isolating dielectric (dielectric constant

$\varepsilon$ ) is considered. The potential due to a point charge $Q$ located at $\vec{r}^{\prime}$ is given by:

$$
\Phi(\vec{r})=\frac{Q}{\varepsilon} G\left(\vec{r}, \vec{r}^{\prime}\right)
$$

that due to an electric charge distribution $\rho_{e}(\vec{r})$ by

$$
\Phi(\vec{r})=\frac{1}{\varepsilon} \iiint d V^{\prime} G\left(\vec{r}, \vec{r}^{\prime}\right) \rho_{e}\left(\vec{r}^{\prime}\right)
$$

$G\left(\vec{r}, \vec{r}^{\prime}\right)$ is the Green's function solving the inhomogeneous potential equation with a unit source:

$$
\Delta G\left(\vec{r}, \vec{r}^{\prime}\right)=-\delta\left(\vec{r}-\vec{r}^{\prime}\right)
$$

fulfilling the same boundary conditions as the potential. The latter will be given below with the specific representations.

\subsection{Green's Function for Free Space}

In a homogeneous medium the potential and the Green's function fulfil the following boundary condition

$$
\vec{r} \rightarrow \infty: \Phi=0, \quad G=0
$$

The latter is given by:

$$
G\left(\vec{r}, \vec{r}^{\prime}\right)=\frac{1}{4 \pi} \frac{1}{R}
$$

with

$$
\begin{aligned}
& R^{2}=\left|\vec{r}-\vec{r}^{\prime}\right|^{2}=\left(x-x^{\prime}\right)^{2}+\left(y-y^{\prime}\right)^{2}+\left(z-z^{\prime}\right)^{2}= \\
& =\rho^{2}-2 \rho \rho^{\prime} \cos \left(\phi-\phi^{\prime}\right)+\rho^{\prime 2}+\left(z-z^{\prime}\right)^{2}= \\
& =\quad P^{2}+\left(z-z^{\prime}\right)^{2} \text {. }
\end{aligned}
$$

The first line is in rectangular coordinates, the second and the third line in cylindrical coordinates $\rho, \phi, z$.

\subsection{Green's Function for an Infinite Plane Condensor}

In this geometry it is no longer possible to represent the Green's function by a closed analytical expression. One must use superpositions of particular solutions of the homogeneous potential equation. These may be infinite series or integrals. Such solutions suffer from the "memory" of the Green's function. There are lines or surfaces passing through the source point, on which these representations display a singular behaviour even if the field point $\vec{r}$ and the source point $\vec{r}^{\prime}$ are still different so that the Green's function is finite. 
In rectangular coordinates $x, y, z$ and in cylindrical coordinates $\rho, \phi, z$ eq.(3) reads:

$$
\begin{aligned}
{\left[\frac{\partial^{2}}{\partial x^{2}}+\frac{\partial^{2}}{\partial y^{2}}+\frac{\partial^{2}}{\partial z^{2}}\right] G\left(x, y, z ; x^{\prime}, y^{\prime}, z^{\prime}\right) } & =-\delta\left(x-x^{\prime}\right) \delta\left(y-y^{\prime}\right) \delta\left(z-z^{\prime}\right) \\
{\left[\frac{\partial^{2}}{\partial \rho^{2}}+\frac{1}{\rho} \frac{\partial}{\partial \rho}+\frac{1}{\rho^{2}} \frac{\partial^{2}}{\partial \phi^{2}}+\frac{\partial^{2}}{\partial z^{2}}\right] G\left(\rho, \phi, z ; \rho^{\prime}, \phi^{\prime}, z^{\prime}\right) } & =-\frac{\delta\left(\rho-\rho^{\prime}\right)}{\rho^{\prime}} \delta\left(\phi-\phi^{\prime}\right) \delta\left(z-z^{\prime}\right) .
\end{aligned}
$$

The solutions of these equations are subject to the following boundary conditions:

$$
\begin{aligned}
z=0, D: & \Phi=0, \quad G=0 \\
\rho \rightarrow \infty: & \Phi=0, \quad G=0
\end{aligned}
$$

Solutions of these equations are represented as Fourier integrals:

$$
G\left(\vec{r}, \vec{r}^{\prime}\right)=\frac{1}{(2 \pi)^{2}} \iint_{-\infty}^{\infty} d k_{x} d k_{y} e^{i\left[k_{x}\left(x-x^{\prime}\right)+k_{y}\left(y-y^{\prime}\right)\right]} g\left(\kappa ; z, z^{\prime}\right)
$$

Inserting the Fourier integral representation of the delta distributions $\delta\left(x-x^{\prime}\right) \delta\left(y-y^{\prime}\right)$ and the above integral representation into eqs.(7) gives the following differential equation for the amplitude $g\left(\kappa ; z, z^{\prime}\right)$ :

$$
\left[\frac{d^{2}}{d z^{2}}-\kappa^{2}\right] g\left(\kappa ; z, z^{\prime}\right)=-\delta\left(z-z^{\prime}\right)
$$

The homogeneous equation corresponding to the above equation is called the one-dimensional diffusion equation. In the z-direction the amplitude $g$ is subject to the same boundary conditions as $G$. The solutions fulfilling all these equations are given below. It turns out that the function $g\left(\kappa ; z, z^{\prime}\right)$ depends only on $\kappa=\sqrt{k_{x}^{2}+k_{y}^{2}}$, so it is convenient to introduce cylindrical coordinates $\rho, \phi, z$ in coordinate space and polar coordinates in $k$-space:

$$
\begin{aligned}
\kappa^{2}= & k_{x}^{2}+k_{y}^{2}, \quad k_{x}=\kappa \cos \psi, \quad k_{y}=\kappa \sin \psi ; \\
P^{2}= & \left(x-x^{\prime}\right)^{2}+\left(y-y^{\prime}\right)^{2}=\rho^{2}+\rho^{\prime 2}-2 \rho \rho^{\prime} \cos \left(\phi-\phi^{\prime}\right) . \\
& k_{x}\left(x-x^{\prime}\right)+k_{y}\left(y-y^{\prime}\right)=\kappa P \cos \left(\phi-\phi^{\prime}-\psi\right) .
\end{aligned}
$$

Using these transformations and Sommerfeld's integral representation of the Bessel functions ([5],[6], §19, [7], p. 20)

$$
2 \pi J_{0}(\kappa P)=\int_{0}^{2 \pi} d \psi e^{i \kappa P \cos \left(\phi-\phi^{\prime}-\psi\right)}
$$

the above representation is rewritten as:

$$
G\left(\vec{r}, \vec{r}^{\prime}\right)=\frac{1}{(2 \pi)} \int_{0}^{\infty} \kappa d \kappa J_{0}(\kappa P) g\left(\kappa ; z, z^{\prime}\right) .
$$

Different representations are found by solving eq.(11) in different ways. 
Besides the closed expression (5) the following integral representation of this Green's function may be given. Below this will be needed to represent a part of the potential of a unit source in a plane condensor; it is

$$
\begin{aligned}
G\left(\vec{r}, \vec{r}^{\prime}\right) & =\frac{1}{(2 \pi)^{2}} \iint_{-\infty}^{\infty} d k_{x} d k_{y} e^{i\left[k_{x}\left(x-x^{\prime}\right)+k_{y}\left(y-y^{\prime}\right)\right]} \frac{e^{-\kappa\left|z-z^{\prime}\right|}}{2 \kappa} \\
& =\frac{1}{(4 \pi)} \int_{0}^{\infty} d \kappa J_{0}(\kappa P) e^{-\kappa\left|z-z^{\prime}\right|} .
\end{aligned}
$$

Equating the last line with (5) gives Sommerfeld's integral ([6], §31 , [7], pp. 384, 416):

$$
\frac{1}{\sqrt{P^{2}+\left(z-z^{\prime}\right)^{2}}}=\int_{0}^{\infty} d \kappa J_{0}(\kappa P) e^{-\kappa\left|z-z^{\prime}\right|} .
$$

The above integral representations are found from the solution of eq.(11) with the interval of definition extending to infinity on both sides so that $g$ must also fulfil the boundary condition

$$
|z| \rightarrow \infty: g=0 \text {. }
$$

which is satisfied by [8]

$$
g\left(\kappa ; z, z^{\prime}\right)=\frac{e^{-\kappa\left|z-z^{\prime}\right|}}{2 \kappa}
$$

\subsubsection{Eigenfunction Expansion of the Green's Function}

The homogeneous equation belonging to eq.(11) has no eigenfunctions. So those of the corresponding Helmholz equation are used [9]:

$$
\begin{aligned}
& {\left[\frac{d^{2}}{d z^{2}}+k^{2}\right] g_{H}\left(\kappa ; z, z^{\prime}\right)=-\delta\left(z-z^{\prime}\right) ;} \\
& g_{H}\left(\kappa ; z, z^{\prime}\right)=\frac{2}{D} \sum_{n=1}^{\infty} \frac{\sin \left(k_{n} z / D\right) \sin \left(k_{n} z^{\prime} / D\right)}{k_{n}^{2}-k^{2}}
\end{aligned}
$$

with $\quad k_{n}=n \pi / D, \quad n=1,2,3, \ldots$.

The substitution $k^{2} \rightarrow-\kappa^{2}$ transforms the Helmholtz (19) into the diffusion equation (11) and the Green's function $g_{H}$ into that we need:

$$
g\left(\kappa ; z, z^{\prime}\right)=\frac{2}{D} \sum_{n=1}^{\infty} \frac{\sin \left(k_{n} z\right) \sin \left(k_{n} z^{\prime}\right)}{k_{n}^{2}+\kappa^{2}} .
$$

This function is inserted into the integral (14). Integrations are done term-wise with the help of

$$
\int_{0}^{\infty} d \kappa \frac{\kappa J_{0}(\kappa P)}{\kappa^{2}+k_{n}^{2}}=K_{0}\left(k_{n} P\right)
$$

to give the following eigenfunction expansion of the Green's function:

$$
G\left(\rho, \phi, z ; \rho^{\prime}, \phi^{\prime}, z^{\prime}\right)=\frac{1}{\pi D} \sum_{n=1}^{\infty} \sin \left(k_{n} z\right) \sin \left(k_{n} z^{\prime}\right) K_{0}\left(k_{n} P\right) .
$$


$k_{n}$ is given above in (21). It is obvious that this representation does not exist on the line $P=0$ passing through the source point. Convergence will be slow near to this line. For sufficiently large values of $P$, the convergence will be excellent due to the exponential decay of the modified Bessel function $K_{0}$ [5]. An example displaying the dependence of the sum on the number of terms is given in Fig.4 .

\subsubsection{An Integral Representation for the Green's Function}

Eq.(11) with the boundary conditions (8) is solved by the method of particular integrals. This gives [9], [10]:

$$
g\left(\kappa ; z, z^{\prime}\right)=\frac{\sinh \left[\kappa\left(D-z_{>}\right)\right] \sinh \left[\kappa z_{<}\right]}{\kappa \sinh (\kappa D)}
$$

with

$$
z_{>}=\max \left(z, z^{\prime}\right), \quad z_{<}=\min \left(z, z^{\prime}\right) .
$$

This gives the following representation of the Green's function for the condensor comprising one layer:

$$
G\left(\rho, \phi, z ; \rho^{\prime}, \phi^{\prime}, z^{\prime}\right)=\frac{1}{2 \pi} \int_{0}^{\infty} d \kappa J_{0}(\kappa P) \frac{\sinh \left[\kappa\left(D-z_{>}\right)\right] \sinh \left[\kappa z_{<}\right]}{\sinh (\kappa D)} .
$$

The above integral may be transformed into one extending over the whole real axis, which may be evaluated by Cauchy's residue theorem. This leads again to the series (23).

\subsubsection{Convergence Properties of the Integral Representation}

The asymptotic behaviour of the Bessel function provides a factor $1 / \sqrt{\kappa}$ for the integrand. Together with the oscillatory behaviour of the Bessel function this leads to convergence, but to

one that is too slow to be of any use in numerical evaluations. So it is the ratio of hyperbolic functions, which may induce good convergence. This ratio is rewritten as:

$$
2 h\left(\kappa ; z, z^{\prime}\right)=\frac{e^{-\kappa\left(z_{>}-z_{<}\right)}-e^{-\kappa\left(z_{>}+z_{<}\right)}-e^{-\kappa\left(2 D-z_{>}-z_{<}\right)}+e^{-\kappa\left(2 D-z_{>}+z_{<}\right)}}{1-e^{-2 \kappa D}} .
$$

From the first exponential in the numerator it is seen that equal values of $z$ and $z^{\prime}$ spoil the convergence inducing property of this ratio, s. Fig.3. So in the plane $z=z^{\prime}$ the integral representation of the Green's function conserves the "memory" of the singularity $\vec{r}=\vec{r}^{\prime}$. But this memory effect of $g\left(\kappa, z, z^{\prime}\right)$ may be cured by subtracting a simple term having the same asymptotic behaviour, i.e. we introduce

$$
\begin{aligned}
g_{r}\left(\kappa ; z, z^{\prime}\right) & :=2 h\left(\kappa, z, z^{\prime}\right)-e^{-\kappa\left(z_{>}-z_{<}\right)}= \\
& =-\frac{e^{-\kappa\left(z_{>}+z_{<}\right)}+e^{-\kappa\left(2 D-z_{>}-z_{<}\right)}-e^{-\kappa\left(2 D-z_{>}+z_{<}\right)}-e^{-\kappa\left(2 D+z_{>}-z_{<}\right)}}{1-e^{-2 \kappa D}} .
\end{aligned}
$$

The integral over the subtrahend is evaluated by Sommerfeld's integral (17). This gives the following new representation of the Green's function:

$$
G\left(\rho, \phi, z ; \rho^{\prime}, \phi^{\prime}, z^{\prime}\right)=\frac{1}{4 \pi \sqrt{P^{2}+\left(z-z^{\prime}\right)^{2}}}+\frac{1}{4 \pi} \int_{0}^{\infty} d \kappa J_{0}(\kappa P) g_{r}\left(\kappa ; z, z^{\prime}\right) .
$$



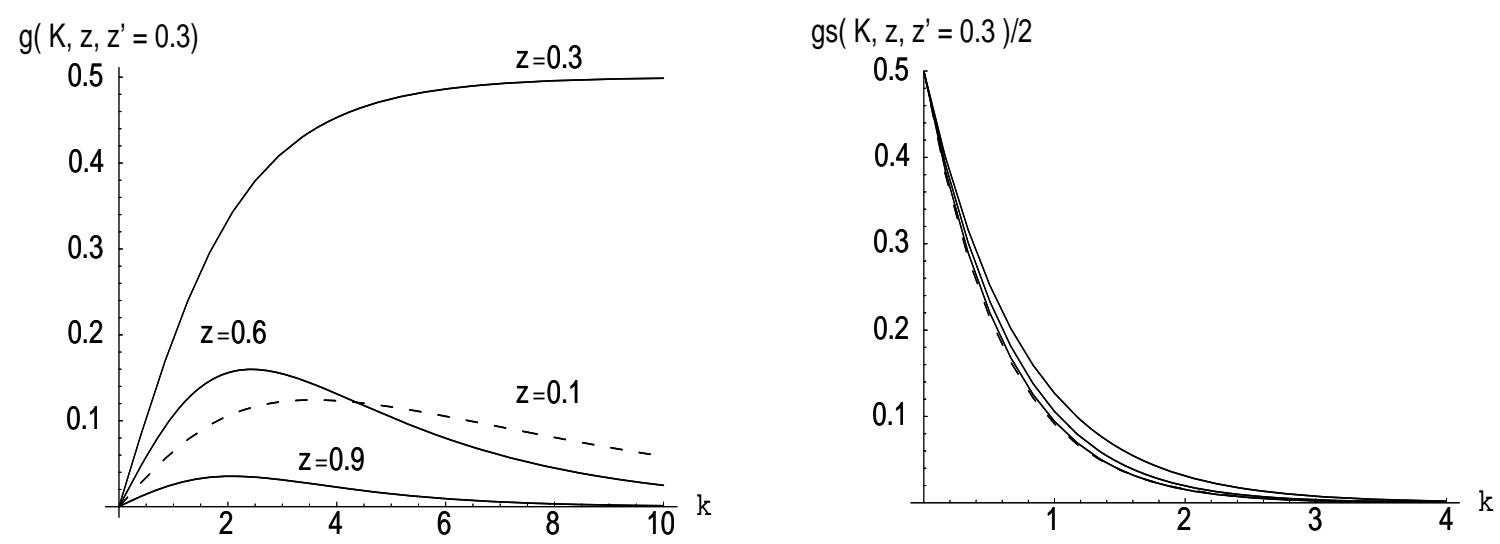

Figure 3: The behaviour of the amplitudes $g\left(\kappa, z, z^{\prime}\right)$, eq.(24), and $g_{s}\left(\kappa, z, z^{\prime}\right) / 2$, eq.(30), for increasing integration variable $\kappa . z^{\prime}=0.3 ; z=0.1,0.3,0.6,0.9$. Note the different scales of the abscissae !

Inspection of $g_{r}$ in Eq.(28) shows that not all dangerous terms have been removed. In fact, $g_{r}$ contains exponentials, whose exponents become zero for $z=z^{\prime}=0\left(e^{-\kappa(z>+z<)}\right)$ or $z=z^{\prime}=$ $D \quad\left(e^{-\kappa\left(2 D-z_{>}-z_{<}\right)}\right)$. These troubles have a physical origin, again a memory effect. In these two cases an image charge obtained by reflecting the primary charge at a metallic boundary is in the same plane (just this boundary) as the primary charge. Both these exponentials must be subtracted from $g_{r}$ to give $g_{s}$ and the corresponding correction terms must be added in front of the integral as this was done just before. So the final form of the modified integral representation of the Green's function of a plane condensor filled with a homogeneous dielectric is:

$$
\begin{aligned}
G\left(\rho, \phi, z ; \rho^{\prime}, \phi^{\prime}, z^{\prime}\right) & =\frac{1}{4 \pi \sqrt{P^{2}+\left(z-z^{\prime}\right)^{2}}}-\frac{1}{4 \pi \sqrt{P^{2}+\left(z+z^{\prime}\right)^{2}}} \\
& -\frac{1}{4 \pi \sqrt{P^{2}+\left(2 D-z-z^{\prime}\right)^{2}}} \\
& +\frac{1}{4 \pi} \int_{0}^{\infty} d \kappa J_{0}(\kappa P) g_{s}\left(\kappa ; z, z^{\prime}\right)
\end{aligned}
$$

with

$$
\begin{aligned}
& g_{s}\left(\kappa, z, z^{\prime}\right)=g_{r}\left(\kappa, z, z^{\prime}\right)+e^{-\kappa\left(z_{>}+z_{<}\right)}+e^{-\kappa\left(2 D-z_{>}-z_{<}\right)} \\
& =\frac{e^{-\kappa\left(2 D-z+z^{\prime}\right)}+e^{-\kappa\left(2 D+z-z^{\prime}\right)}-e^{-\kappa\left(2 D+z+z^{\prime}\right)}-e^{-\kappa\left(4 D-z-z^{\prime}\right)}}{1-e^{-2 \kappa D}} .
\end{aligned}
$$

This modified integral representation works fine since $g_{s}$ decreases rather fast with increasing $\kappa$, and this quite independent of the arguments $P, z$ and $z^{\prime}$ as shown in Fig.3.

\subsubsection{Comparison of the Two Representations}

Here the cured integral representation, (29), is compared to the series representation, (23). This is done for specific values for the arguments of the Green's function given in Fig.4. All the evaluations are done in Mathematica [4], Version 4.0.1, on a Macintosh PowerPC 8500 with $300 \mathrm{MHz}$ under system Mac OS D1-8.6. The cured integral representation gives reliable values 
$G_{n}$ with a precision better than $10^{-8}$ for an upper integration limit $\kappa=8$ (in place of $\infty$ ). The time needed for one integration is $0.067 \mathrm{~s}$. As many terms of the series were taken as necessary to give a value differing from $G_{n}$ no more than $5 \times 10^{-7}$.

In the uppermost part of Fig.4 the value of the Green's function is plotted versus the distance $\rho$ from the singular line $\rho^{\prime}=0$. In the center the number of terms needed to attain the wanted accuracy is given. The lowest diagram shows the time needed for calculating and summing these terms. A program for calculating the Green's function may either rely completely on the integral representation but will then incur somewhat longer computing times than one based on a strategy with branching between the two representations. The boundary between the two domains must be found beforehand by a careful analysis varying the values of $P, z$ and $z^{\prime}$. In condensors with more than one layer the series expansion incurs additional disadvantages. 

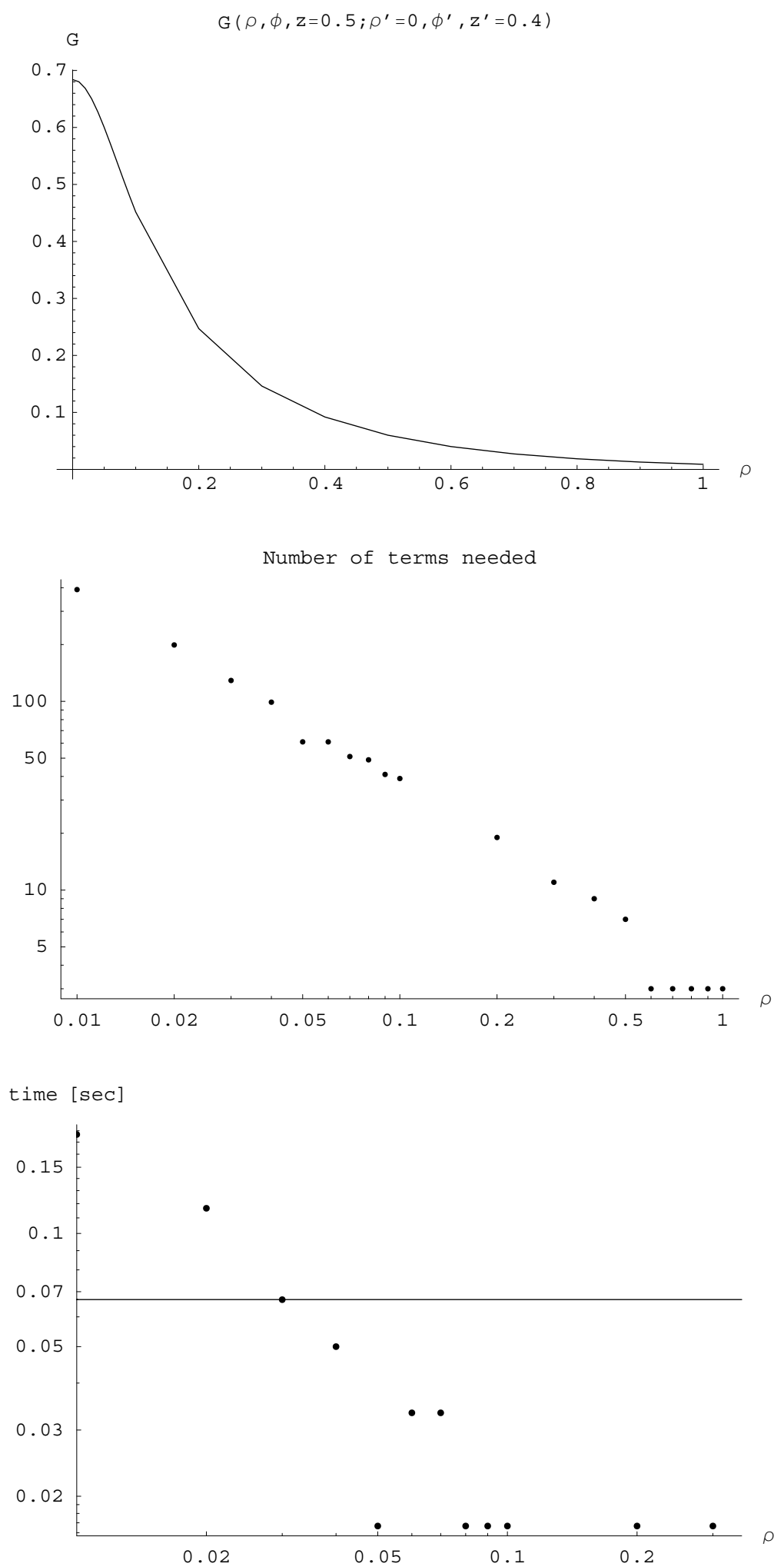

Figure 4: Upper picture: Values of the Green's function $G$ versus polar radius $\rho$. The charge is located in the $z$-axis; $\rho^{\prime}=0, z^{\prime}=0.4 ; z=0.5$. Values were computed only for discrete values of $P$ and connected by straight lines. Center: The number of terms of the series (23) needed for an error $<5 \times 10^{-7}$. Lowest picture: Time needed for the evaluation of the series (Macintosh PowerPC 8500, $300 \mathrm{MHz}$ ). The horizontal line gives the time needed for the numeric evaluation of the cured integral. 8 


\subsection{The Weighting Field of a Strip Electrode}

As an application the Green's function derived above is used to calculate the weighting field of an electrode having the shape of a strip as they are used in RPC's. Three assumptions are made to simplify the analysis: i) the strip is infinitely thin; ii) it has infinite length; this transforms the problem into a two-dimensional one; (then one may use conformal maps [10]); iii) the gaps between the strips are disregarded.

The current induced on an electrode $i$ by a point charge $q$ moving with velocity $\vec{v}$ may be calculated from Ramo's theorem [11] and may be expressed as [12]:

$$
I_{i}=-q \vec{v} \cdot \frac{\vec{E}_{i}(\vec{r})}{V_{i}}=q \vec{v} \cdot \frac{1}{V_{i}} \vec{\nabla} \Phi_{i}(\vec{r}),
$$

where $V_{i}$ is the voltage applied to the electrode $i$ generating the electric field $\vec{E}_{i}(\vec{r})$ in the absence of the charge $q$ and having all the other electrodes grounded. $\vec{E}_{i}(\vec{r})$ is called the weighting field [13]. We assume that it may be derived from a scalar potential $\Phi_{i}(\vec{r})$, which we shall call the weighting potential. Under the simplifying assumptions just listed this potential is computed by assuming that a constant voltage $V_{1}$ is impressed on the strip $-w / 2 \leq x \leq w / 2,-\infty \leq$ $y \leq \infty$ of the continuous metallic electrode at $z=0$; while the complementary part of this electrode and the other electrode are grounded. With the help of Greens second theorem, with the differential equation for the Green's function, (3), with the Laplace equation for the potential and with the boundary condition (8) the following representation for the wanted weighting potential of electrode 1 is found:

$$
\Phi_{1}(\vec{r})=\left.V_{1} \int_{-w / 2}^{w / 2} d x^{\prime} \int_{-\infty}^{\infty} d y^{\prime} \frac{\partial G\left(\vec{r} ; x^{\prime}, y^{\prime}, z^{\prime}\right)}{\partial z^{\prime}}\right|_{z^{\prime}=0} .
$$

All computations are done in Cartesian coordinates. Thus the integral representation (10) is used. The integration over $y^{\prime}$ gives $2 \pi$ times a delta distribution $\delta\left(k_{y}\right)$. Note that the subsequent integration over $k_{y}$ reduces $\kappa=\sqrt{k_{x}^{2}+k_{y}^{2}}$ to $\kappa=\left|k_{x}\right|$ ! Then the integration over $x^{\prime}$ is done and the symmetry of the resulting integrand is used to reduce the range of integration over $\kappa$ to the positive part of the real axis. This gives for the weighting potential:

$$
\Phi_{1}(x, z)=\left.V_{1} \frac{2}{\pi} \int_{0}^{\infty} d \kappa \cos (\kappa x) \sin \left(\kappa \frac{w}{2}\right) \frac{1}{\kappa} \frac{\partial g\left(\kappa ; z, z^{\prime}\right)}{\partial z^{\prime}}\right|_{z^{\prime}=0} .
$$

The amplitude function $g\left(\kappa ; z, z^{\prime}\right)$ is taken from (24). So we get for $z>z^{\prime}=0$ :

$$
\begin{aligned}
\Phi_{1}(x, z)= & V_{1} \frac{2}{\pi} \int_{0}^{\infty} d \kappa \cos (\kappa x) \sin \left(\kappa \frac{w}{2}\right) \frac{\sinh (\kappa(D-z))}{\kappa \sinh (\kappa D)} \\
= & \frac{V_{1}}{\pi}\left[\arctan \left(\cot \left(\frac{z \pi}{2 D}\right) \tanh \left(\pi \frac{x+w / 2}{2 D}\right)\right)\right. \\
& \left.\quad-\arctan \left(\cot \left(\frac{z \pi}{2 D}\right) \tanh \left(\pi \frac{x-w / 2}{2 D}\right)\right)\right] .
\end{aligned}
$$

The two components of the weighting field are derived from this as:

$$
E_{1 x}=-\frac{\partial \Phi_{1}}{\partial x}=V_{1} \frac{2}{\pi} \int_{0}^{\infty} d \kappa \sin (\kappa x) \sin \left(\kappa \frac{w}{2}\right) \frac{\sinh (\kappa(D-z))}{\sinh (\kappa D)}
$$




$$
\begin{aligned}
& =V_{1} \frac{1}{2 D}\left[\frac{\sin \left(\frac{z \pi}{D}\right)}{\cosh \left(\pi \frac{x-w / 2}{D}\right)-\cos \left(\frac{z \pi}{D}\right)}-\frac{\sin \left(\frac{z \pi}{D}\right)}{\cosh \left(\pi \frac{x+w / 2}{D}\right)-\cos \left(\frac{z \pi}{D}\right)}\right] \\
E_{1 z} & =-\frac{\partial \Phi_{1}}{\partial z}=V_{1} \frac{2}{\pi} \int_{0}^{\infty} d \kappa \cos (\kappa x) \sin \left(\kappa \frac{w}{2}\right) \frac{\cosh (\kappa(D-z))}{\sinh (\kappa D)} \\
& =-V_{1} \frac{1}{2 D}\left[\frac{\sinh \left(\pi \frac{x-w / 2}{D}\right)}{\cosh \left(\pi \frac{x-w / 2}{D}\right)-\cos \left(\frac{z \pi}{D}\right)}-\frac{\sinh \left(\pi \frac{x+w / 2}{D}\right)}{\cosh \left(\pi \frac{x+w / 2}{D}\right)-\cos \left(\frac{z \pi}{D}\right)}\right] .
\end{aligned}
$$

The integrals over $\kappa$ above were evaluated by decomposing the product of the trigonometric functions and the resulting integrals were found in tables [14].

\subsubsection{Weighting Field Computed by Conformal Map}

The potential (34) may serve as a starting point for finding a conformal map, which permits one to compute and plot equipotential and field lines rather easily. $x$ and $z$ are combined to a complex variable $Z:=x+i z . \Phi_{1}(x, z)=\Phi_{r}(x, z)$ is a harmonic function in its two arguments, so it may be regarded as the real part of a complex function $\Phi_{w}(Z)$. The imaginary part of this function is found via the Cauchy-Riemann equations:

$$
\begin{aligned}
& \Phi_{i}(x, z)=\frac{V_{1}}{2 \pi}[\quad \log [\cosh (\pi(x+w / 2) / D)-\cos (\pi y / D)] \\
& -\log [\cosh (\pi(x-w / 2) / D)-\cos (\pi y / D)]] .
\end{aligned}
$$

The corresponding complex potential

$$
\begin{aligned}
\Phi_{w}(Z) & =\Phi_{r}(x, z)+i \Phi_{i}(x, z)= \\
& =\frac{V_{1}}{2 \pi} \log \left[\sinh \left[\pi \frac{x+w / 2-i z}{2 D}\right] / \sinh \left[\pi \frac{x-w / 2-i z}{2 D}\right]\right] \\
& :=\Psi(W)=-i \frac{V_{1}}{\pi} W
\end{aligned}
$$

is equated to that, $\Psi(W)$, of the uniform field within a plane condensor whose electrodes are at potential $0, V_{1}$ respectively. Then the function

$$
\begin{aligned}
Z=x+i z=f(W) & =f(U+i V) \\
& =\frac{D}{\pi} \log \left[\frac{e^{W} \alpha^{2}-1}{e^{W}-\alpha^{2}}\right]^{*}
\end{aligned}
$$

with

$$
\alpha=e^{\pi w /(4 D)}
$$

maps this plane condensor onto the configuration with the strip electrode.

The images of the straight lines $V=$ const. ( $U=$ const.) are the equipotential (field) lines. The curves so obtained are shown in Fig.5. 


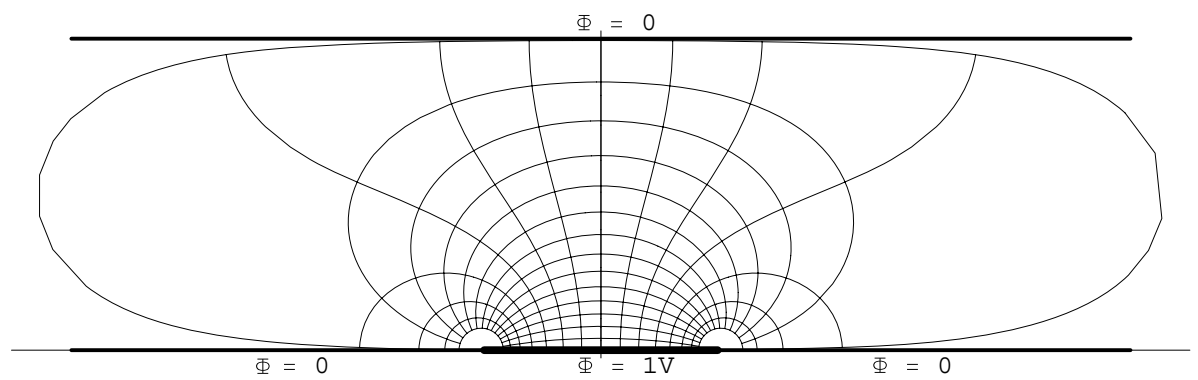

Figure 5: Equipontial and field lines in a plane condensor with an anode strip at 1 Volt, while the remaining part of that electrode and the other electrode are grounded. 


\section{Green's Function for the Three-Layer Problem}

In this section the plane condensor comprising three homogeneous isolating dielectric layers is treated. The configuration is shown in Fig.1. The electrodes are at $z=-q<0$ and at $z=p>g>0$. The gas gap corresponds to layer $2(0 \leq z \leq g)$ with a dielectric constant $\varepsilon_{2}$, the two panes $(-q<z<0),(g<z<p)$ respectively have a dielectric constant $\varepsilon_{1}, \varepsilon_{3}$ respectively. The $\varepsilon_{i}$ 's represent the full dielectric constants, i.e. they are $\varepsilon_{0}$ times the relative dielectric constant.

In the first subsections some general derivations concerning the potential equation containing variable dielectric constant and the corresponding Green's function are given. This Green's function is used to relate the potential to the electric charge distribution $\rho_{e}(\vec{r})$. Then the expressions for the Green's function of the three-layer problem are derived. Thereafter the exponentials dangerous for the convergence of these expressions are identified and neutralized.

\subsection{Green's Theorem and Functions for Variable Dielectric Constant}

At first one starts from a dielectric constant, which is a differentiable function of the space coordinate $z$, so $\varepsilon=\varepsilon(z)$. Then Poisson's equation is:

$$
\nabla \cdot(\varepsilon(z) \nabla) \Phi(\vec{r})=-\rho_{e}(\vec{r})
$$

Two ideally conducting planes bound the space at $z=p,-q$. The domain between these extends

to infinity; there, too, the potential and so the Green's function must be zero:

$$
\begin{aligned}
z=p,-q: & \Phi=0, \quad G=0 \\
\rho \rightarrow \infty: & \Phi=0, \quad G=0
\end{aligned}
$$

Green's function is a solution of the following equation:

$$
\nabla \cdot(\varepsilon(z) \nabla) G\left(\vec{r}, \vec{r}^{\prime}\right)=-\delta\left(\vec{r}-\vec{r}^{\prime}\right)
$$

Note that the above expression is self-adjoint, so the Green's function is symmetric in its arguments:

$$
G\left(\vec{r}, \vec{r}^{\prime}\right)=G\left(\vec{r}^{\prime}, \vec{r}\right) .
$$

The following generalized form of Green's second theorem is needed for the above differential operators:

$$
\begin{aligned}
& \iiint d V^{\prime}\left[G\left(\vec{r}^{\prime}, \vec{r}\right) \nabla^{\prime} \cdot\left(\varepsilon\left(z^{\prime}\right) \nabla^{\prime}\right) \Phi\left(\vec{r}^{\prime}\right)-\Phi\left(\vec{r}^{\prime}\right) \nabla^{\prime} \cdot\left(\varepsilon\left(z^{\prime}\right) \nabla^{\prime}\right) G\left(\vec{r}^{\prime}, \vec{r}\right)\right]= \\
& =\iint d S^{\prime}\left[G\left(\vec{r}, \vec{r}^{\prime}\right) \varepsilon\left(z^{\prime}\right) \frac{\partial \Phi\left(\vec{r}^{\prime}\right)}{\partial n^{\prime}}-\Phi\left(\vec{r}^{\prime}\right) \varepsilon\left(z^{\prime}\right) \frac{\partial G}{\partial n^{\prime}}\right] d S^{\prime}
\end{aligned}
$$

The surface integral on the rhs of the above equation is zero due to the homogeneous boundary conditions (43) and (44). Eqs. (42) and (45) are inserted into the above equation and are evaluated to give:

$$
\Phi(\vec{r})=\iiint d V^{\prime} G\left(\vec{r}, \vec{r}^{\prime}\right) \rho_{e}\left(\vec{r}^{\prime}\right) .
$$




\subsection{Specialization to Three Homogeneous Layers}

Now the dielectric constant has constant values $\varepsilon_{1}, \varepsilon_{2}, \varepsilon_{3}$ as shown in Fig.1. Then the above integral becomes:

$$
\begin{aligned}
\Phi_{i}(\vec{r}) & =\iiint_{z^{\prime}=-q}^{0} d V^{\prime} G_{i 1}\left(\vec{r}, \vec{r}^{\prime}\right) \rho_{e 1}\left(\vec{r}^{\prime}\right) \\
& +\iiint_{z^{\prime}=0}^{g} d V^{\prime} G_{i 2}\left(\vec{r}, \vec{r}^{\prime}\right) \rho_{e 2}\left(\vec{r}^{\prime}\right) \\
& +\iiint_{z^{\prime}=g}^{p} d V^{\prime} G_{i 3}\left(\vec{r}, \vec{r}^{\prime}\right) \rho_{e 3}\left(\vec{r}^{\prime}\right) ; \quad i=1,2,3 .
\end{aligned}
$$

The differential equation for the Green's function (45) decomposes into a set of 9 differential equations:

$$
\Delta G_{i k}\left(\vec{r}, \vec{r}^{\prime}\right)=-\frac{1}{\varepsilon_{i}} \delta_{i k} \delta\left(\vec{r}-\vec{r}^{\prime}\right), \quad i, k=1,2,3
$$

(no summation over repeated subscripts !) subject to the following boundary and continuity conditions:

$$
\begin{aligned}
z=-q: & G_{1 k}=0, & k=1,2,3 ; \\
z=p: & G_{3 k}=0, & k=1,2,3 ; \\
\rho \rightarrow \infty: & G_{i k}=0, & i, k=1,2,3 ;
\end{aligned}
$$

and continuity conditions:

$$
\begin{array}{llll}
z=0: & G_{1 k}=G_{2 k}, & \varepsilon_{1} \frac{\partial G_{1 k}}{\partial z}=\varepsilon_{2} \frac{\partial G_{2 k}}{\partial z}, & k=1,2,3 \\
z=g: & G_{2 k}=G_{3 k}, & \varepsilon_{2} \frac{\partial G_{2 k}}{\partial z}=\varepsilon_{3} \frac{\partial G_{3 k}}{\partial z}, & k=1,2,3 .
\end{array}
$$

Note that the Green's functions is a scalar in spite of the subscripts; but different functions represent it depending on the location of the arguments in the various layers. This dependencies are made explicit by these subscripts; the first (second) one gives the layer containing the point of observation (source point) according to the Fig. 1 . We call these various functions $G_{i k}\left(\vec{r}, \vec{r}^{\prime}\right)$ pieces and shall use the adjectives diagonal (off-diagonal) for equal (non-equal) subscripts.

\subsection{Derivation of the Green's Function.}

The Green's function is represented as a Fourier integral in the two horizontal variables $x$ and $y$ :

$$
G_{i k}\left(\vec{r}, \vec{r}^{\prime}\right)=\frac{1}{(2 \pi)^{2}} \iint_{-\infty}^{\infty} d k_{x} d k_{y} e^{i\left[k_{x}\left(x-x^{\prime}\right)+k_{y}\left(y-y^{\prime}\right)\right]} g_{i k}\left(\kappa ; z, z^{\prime}\right) .
$$

Inserting the Fourier integral representation of the delta distributions $\delta\left(x-x^{\prime}\right) \delta\left(y-y^{\prime}\right)$ and the above integral representation into eqs.(50) gives the following system of differential equations for the amplitudes $g_{i k}\left(\kappa ; z, z^{\prime}\right)$ :

$$
\left(\frac{d^{2}}{d z^{2}}-\kappa^{2}\right) g_{i k}\left(\kappa ; z, z^{\prime}\right)=-\delta_{i k} \delta\left(z-z^{\prime}\right)
$$

subject to the same boundary and continuity conditions as the $G_{i k}$, eqs.(51) to (53). The solutions fulfilling all these equations are given below. It turns out that the function $g_{i k}\left(\kappa ; z, z^{\prime}\right)$ depends only on $\kappa=\sqrt{k_{x}^{2}+k_{y}^{2}}$. So it is convenient to introduce cylindrical coordinates $\rho, \phi, z$ 
in coordinate space and polar coordinates in $k$-space as in eqs.(6) and (12). Using these transformations and Sommerfeld's integral representation of the Bessel functions (13) the above representation is rewritten as:

$$
G_{i k}\left(\vec{r}, \vec{r}^{\prime}\right)=\frac{1}{(2 \pi)} \int_{0}^{\infty} \kappa d \kappa J_{0}(\kappa P) g_{i k}\left(\kappa ; z, z^{\prime}\right) .
$$

The solutions for the amplitudes have been found, transformed and simplified with the help of Mathematica [4] in lengthy calculations. The diagonal amplitudes are written as :

$$
g_{i i}\left(\kappa ; z, z^{\prime}\right)=\frac{e^{-\kappa\left|z-z^{\prime}\right|}}{2 \varepsilon_{i} \kappa}+\frac{N_{i i}\left(\kappa ; z, z^{\prime}\right)}{2 \varepsilon_{i} \kappa D(\kappa)} ;
$$

and the off-diagonal amplitudes as:

$$
g_{i k}\left(\kappa ; z, z^{\prime}\right)=\frac{N_{i k}\left(\kappa ; z, z^{\prime}\right)}{\kappa D(\kappa)} .
$$

All $g_{i k}$ have the same denominator $D(\kappa)$ :

$$
\begin{aligned}
D(\kappa)= & c_{0}\left(1-e^{-2 \kappa(p+q)}\right)-\left(\varepsilon_{1}-\varepsilon_{2}\right)\left(\varepsilon_{2}+\varepsilon_{3}\right)\left(e^{-2 \kappa p}-e^{-2 \kappa q}\right) \\
& -\left(\varepsilon_{1}+\varepsilon_{2}\right)\left(\varepsilon_{2}-\varepsilon_{3}\right)\left(e^{-2 \kappa(p-g)}-e^{-2 \kappa(g+q)}\right) \\
& +\left(\varepsilon_{1}-\varepsilon_{2}\right)\left(\varepsilon_{2}-\varepsilon_{3}\right)\left(e^{-2 \kappa g}-e^{-2 \kappa(p+q-g)}\right)
\end{aligned}
$$

The constant $c_{0}$ gives a constant finite limit of the denominator

$$
c_{0}=\left(\varepsilon_{1}+\varepsilon_{2}\right)\left(\varepsilon_{2}+\varepsilon_{3}\right)
$$

for $\kappa \rightarrow \infty$ as long as none of the three layer has zero width. 


\section{The various numerators are:}

$$
\begin{aligned}
& 2 \varepsilon_{1} N_{11}\left(\kappa ; z, z^{\prime}\right)= \\
& \left(\varepsilon_{1}+\varepsilon_{2}\right)\left(\varepsilon_{2}+\varepsilon_{3}\right)\left(e^{-\kappa\left(2 p+2 q+z-z^{\prime}\right)}+e^{-\kappa\left(2 p+2 q-z+z^{\prime}\right)}-e^{-\kappa\left(2 p-z-z^{\prime}\right)}-\right. \\
& \left.e^{-\kappa\left(2 q+z+z^{\prime}\right)}\right)+ \\
& \left(\varepsilon_{1}-\varepsilon_{2}\right)\left(\varepsilon_{2}+\varepsilon_{3}\right)\left(e^{\kappa\left(z+z^{\prime}\right)}+e^{-\kappa\left(2 p+2 q+z+z^{\prime}\right)}-e^{-\kappa\left(2 q+z-z^{\prime}\right)}-e^{-\kappa\left(2 q-z+z^{\prime}\right)}\right)+ \\
& \left(\varepsilon_{1}+\varepsilon_{2}\right)\left(\varepsilon_{2}-\varepsilon_{3}\right)\left(e^{-\kappa\left(2 g-z-z^{\prime}\right)}+e^{-2 \kappa\left(2 p+2 q-2 g+z+z^{\prime}\right)}-e^{-2 \kappa\left(2 q+2 g-z+z^{\prime}\right)}-\right. \\
& \left.e^{-2 \kappa\left(2 q+2 g+z-z^{\prime}\right)}\right)+ \\
& \left(\varepsilon_{1}-\varepsilon_{2}\right)\left(\varepsilon_{2}-\varepsilon_{3}\right)\left(e^{-\kappa\left(2 p+2 q-2 g-z+z^{\prime}\right)}+e^{-\kappa\left(2 p+2 q-2 g+z-z^{\prime}\right)}-e^{-\kappa\left(2 p-2 g-z-z^{\prime}\right)}-\right. \\
& \left.e^{-\kappa\left(2 q+2 g+z+z^{\prime}\right)}\right) \\
& \begin{array}{c}
N_{21}\left(\kappa ; z, z^{\prime}\right)= \\
\left(\varepsilon_{2}+\varepsilon_{3}\right)\left(e^{-\kappa\left(z-z^{\prime}\right)}+e^{-\kappa\left(2 p+2 q-z+z^{\prime}\right)}-e^{-\kappa\left(2 p-z-z^{\prime}\right)}-e^{-\kappa\left(2 q+z+z^{\prime}\right)}\right)+
\end{array} \\
& \left(\varepsilon_{2}-\varepsilon_{3}\right)\left(e^{-\kappa\left(2 g-z-z^{\prime}\right)}+e^{-\kappa\left(2 p+2 q-2 g+z+z^{\prime}\right)}-e^{-\kappa\left(2 g+2 q-z+z^{\prime}\right)}-e^{-\kappa\left(2 p-2 g+z-z^{\prime}\right)}\right), \\
& N_{31}\left(\kappa ; z, z^{\prime}\right)=2 \varepsilon_{2}\left(e^{-\kappa\left(z-z^{\prime}\right)}+e^{-\kappa\left(2 p+2 q-z+z^{\prime}\right)}-e^{-\kappa\left(2 p-z-z^{\prime}\right)}-e^{-\kappa\left(2 q+z+z^{\prime}\right)}\right) ; \\
& N_{12}\left(\kappa ; z, z^{\prime}\right)= \\
& \left(\varepsilon_{2}+\varepsilon_{3}\right)\left(e^{\kappa\left(z-z^{\prime}\right)}+e^{-\kappa\left(2 p+2 q+z-z^{\prime}\right)}-e^{-\kappa\left(2 p-z-z^{\prime}\right)}-e^{-\kappa\left(2 q+z+z^{\prime}\right)}\right)+ \\
& \left(\varepsilon_{2}-\varepsilon_{3}\right)\left(e^{-\kappa\left(2 g-z-z^{\prime}\right)}+e^{-\kappa\left(2 p+2 q-2 g+z+z^{\prime}\right)}-e^{-\kappa\left(2 p-2 g-z+z^{\prime}\right)}-e^{-\kappa\left(2 g+2 q+z-z^{\prime}\right)}\right), \\
& 2 \varepsilon_{2} N_{22}\left(\kappa ; z, z^{\prime}\right)= \\
& \left(\varepsilon_{1}+\varepsilon_{2}\right)\left(\varepsilon_{2}+\varepsilon_{3}\right)\left(e^{-\kappa\left(2 p+2 q+z-z^{\prime}\right)}+e^{-\kappa\left(2 p+2 q-z+z^{\prime}\right)}-e^{-\kappa\left(2 p-z-z^{\prime}\right)}-e^{-\kappa\left(2 q+z+z^{\prime}\right)}\right)- \\
& \left(\varepsilon_{1}+\varepsilon_{2}\right)\left(\varepsilon_{2}-\varepsilon_{3}\right)\left(e^{-\kappa\left(2 g+2 q+z-z^{\prime}\right)}+e^{-\kappa\left(2 g+2 q-z+z^{\prime}\right)}-e^{-\kappa\left(2 g-z-z^{\prime}\right)}-e^{-\kappa\left(2 p+2 q-2 g+z+z^{\prime}\right)}\right)- \\
& \left(\varepsilon_{1}-\varepsilon_{2}\right)\left(\varepsilon_{2}+\varepsilon_{3}\right)\left(e^{-\kappa\left(z+z^{\prime}\right)}+e^{-\kappa\left(2 p+2 q-z-z^{\prime}\right)}-e^{-\kappa\left(2 p+z-z^{\prime}\right)}-e^{-\kappa\left(2 p-z+z^{\prime}\right)}\right)+ \\
& \left(\varepsilon_{1}-\varepsilon_{2}\right)\left(\varepsilon_{2}-\varepsilon_{3}\right)\left(e^{-\kappa\left(2 g+2 q-z-z^{\prime}\right)}+e^{-\kappa\left(2 p-2 g+z+z^{\prime}\right)}-e^{-\kappa\left(2 g+z-z^{\prime}\right)}-e^{-\kappa\left(2 g-z+z^{\prime}\right)}\right) \text {, } \\
& N_{32}\left(\kappa ; z, z^{\prime}\right)= \\
& \left(\varepsilon_{1}+\varepsilon_{2}\right)\left(e^{-\kappa\left(z-z^{\prime}\right)}+e^{-\kappa\left(2 p+2 q-z+z^{\prime}\right)}-e^{-\kappa\left(2 p-z-z^{\prime}\right)}-e^{-\kappa\left(2 q+z+z^{\prime}\right)}\right)- \\
& \left(\varepsilon_{1}-\varepsilon_{2}\right)\left(e^{-\kappa\left(z+z^{\prime}\right.}+e^{-\kappa\left(2 p+2 q-z-z^{\prime}\right)}-e^{-\kappa\left(2 p-z+z^{\prime}\right)}-e^{-\kappa(2 q+z-z)}\right) \text {; } \\
& N_{13}\left(\kappa ; z, z^{\prime}\right)=2 \varepsilon_{2}\left(e^{\kappa\left(z-z^{\prime}\right)}+e^{-\kappa\left(2 p+2 q+z-z^{\prime}\right)}-e^{-\kappa\left(2 p-z-z^{\prime}\right)}-e^{-\kappa\left(2 q+z+z^{\prime}\right)}\right), \\
& N_{23}\left(\kappa ; z, z^{\prime}\right)= \\
& \left(\varepsilon_{1}+\varepsilon_{2}\right)\left(e^{\kappa\left(z-z^{\prime}\right)}+e^{-\kappa\left(2 p+2 q+z-z^{\prime}\right)}-e^{-\kappa\left(2 p-z-z^{\prime}\right)}-e^{-\kappa\left(2 q+z+z^{\prime}\right)}\right)- \\
& \left(\varepsilon_{1}-\varepsilon_{2}\right)\left(e^{-\kappa\left(z+z^{\prime}\right)}+e^{-\kappa\left(2 p+2 q-z-z^{\prime}\right)}-e^{-\kappa\left(2 p+z-z^{\prime}\right)}-e^{-\kappa\left(2 q-z+z^{\prime}\right)}\right), \\
& 2 \varepsilon_{3} N_{33}\left(\kappa ; z, z^{\prime}\right)= \\
& \left(\varepsilon_{1}+\varepsilon_{2}\right)\left(\varepsilon_{2}+\varepsilon_{3}\right)\left(e^{-\kappa\left(2 p+2 q+z-z^{\prime}\right)}+e^{-\kappa\left(2 p+2 q-z+z^{\prime}\right)}-e^{-\kappa\left(2 p-z-z^{\prime}\right)}-e^{-\kappa\left(2 q+z+z^{\prime}\right)}\right)- \\
& \left(\varepsilon_{1}-\varepsilon_{2}\right)\left(\varepsilon_{2}+\varepsilon_{3}\right)\left(e^{-\kappa\left(z+z^{\prime}\right)}+e^{-\kappa\left(2 p+2 q-z-z^{\prime}\right)}-e^{-\kappa\left(2 p+z-z^{\prime}\right)}-e^{-\kappa\left(2 p-z+z^{\prime}\right)}\right)-
\end{aligned}
$$




$$
\begin{gathered}
\left(\varepsilon_{1}+\varepsilon_{2}\right)\left(\varepsilon_{2}-\varepsilon_{3}\right)\left(e^{\kappa\left(2 g-z-z^{\prime}\right)}+e^{-\kappa\left(2 g+2 p+2 q-z-z^{\prime}\right)}-e^{-\kappa\left(2 p-2 g-z+z^{\prime}\right)}-e^{-\kappa\left(2 p-2 g+z-z^{\prime}\right)}\right)- \\
\left(\varepsilon_{1}-\varepsilon_{2}\right)\left(\varepsilon_{2}-\varepsilon_{3}\right)\left(e^{-\kappa\left(2 p+2 g-z-z^{\prime}\right)}+e^{\kappa\left(2 g-2 q-z-z^{\prime}\right)}-e^{-\kappa\left(2 p+2 q-2 g-z+z^{\prime}\right)}-\right. \\
\left.-e^{-\kappa\left(2 p+2 q-2 g+z-z^{\prime}\right)}\right)
\end{gathered}
$$

With the help of Sommerfeld's integral (17) the diagonal pieces of the Green's function may be rewritten as:

$$
G_{i i}\left(\vec{r}, \vec{r}^{\prime}\right)=\frac{1}{4 \pi \varepsilon_{i}} \frac{1}{R}+\frac{1}{4 \pi \varepsilon_{i}} \int_{0}^{\infty} \kappa d \kappa J_{0}(\kappa P) \frac{N_{i i}\left(\kappa ; z, z^{\prime}\right)}{D(\kappa)} .
$$

with $R=\sqrt{P^{2}+\left(z-z^{\prime}\right)^{2}}$. By this a slowly convergent part of the integrand has been replaced with a closed expression. Eqs.(56) and (62) together with (58) to (61) give integral representations of the various pieces $G_{i k}\left(\vec{r}, \vec{r}^{\prime}\right)$ of the Green's function $G\left(\vec{r}, \vec{r}^{\prime}\right)$. But these are not yet completely suitable for numeric evaluations.

\subsection{Removal of Slowly Convergent Contributions}

For certain values of the coordinates $z$ and $z^{\prime}$ the convergence of these integrals is very slow; in fact, too slow from the point of view of numerical integration. Confer the discussion in subsect. 2.2.4. As this has been shown in the one-layer case the problem can be remedied by neutralizing these terms in the integrand responsible for this slow decay by subtracting simple terms having the same asymptotic behaviour. The integrals over these simple terms can be evaluated analytically by Sommerfelds integral (17) and the resulting expressions are added to the modified integral such that the sum gives the same result as the original integral. This can be done in such a way that the new integrands converge everywhere and this at a rate much faster than that of the original integral. The price to be paid is a greater number of terms in the new integrands.

This method can be understood in terms of physics. The potential of the problem under consideration could also be expressed as a sum comprising the potential of the original charge and the potentials of several infinite chains of images obtained by reflecting this charge at all interfaces and boundaries and by reflecting these images again and again. This method is not suitable in this case. The infinite series resulting from these chains of images are conditionally and slowly convergent and must be treated with considerable precautions [10], [15]. But from the knowledge about these images we find the explanation of the failures discussed in the previous paragraph. If the image in the adjacent layer and the original charge coincide, then this representation of the solution fails. There is a memory of this hidden in the solution to the effect that the integrals converge very slowly or may even diverge if the source and the observation point are in the same horizontal plane though they are not at the same place. The problem is even more severe for field representations derived from this representations of the potential.

But here there is a remedy, which was described in the first paragraph of this section and demonstrated in sect.2.2.4. This method is applied the Green's function of the three layer condensor in the next subsection.

\subsubsection{Performing the Removal}

In the integral representations (56) and (62) the factor $\kappa$ besides the differential cancel against the same factor occurring in the denominator $D(\kappa) . D(\kappa)$ tends towards the constant $c_{0}$ for 
large $\kappa$. The asymptotic behaviour of the Bessel function provides a factor $1 / \sqrt{\kappa}$; together with the oscillations of the Bessel function the convergence of the integrand is ensured as displayed by the Sommerfeld integral. But the convergence is too slow for numerical purposes if the other factors stay constant. This just happens for some combinations of $z, z^{\prime}, g, p$ and $q$ in the exponentials of the numerator; these combinations will be abbreviated as $z^{(n)}$. Such a term looks like

$$
\frac{c_{i k}^{(n)}}{c_{0}} e^{-\kappa z_{i k}^{(n)}}
$$

in the limit $\kappa \rightarrow \infty$. Their deleterious effect is cured by compensating each dangerous term by subtracting the corresponding asymptotic term given above from the ratio $N_{i k} / D(\kappa)$ :

$$
\begin{aligned}
\frac{R_{i k}}{D(\kappa)} & :=\frac{N_{i k}\left(\kappa ; z, z^{\prime}\right)}{D(\kappa)}-\sum_{n} \frac{c_{i k}^{(n)}}{c_{0}} e^{-\kappa z_{i k}^{(n)}}= \\
& =\frac{N_{i k} c_{0}-\sum_{n} c_{i k}^{(n)} e^{-\kappa z_{i k}^{(n)}} D(\kappa)}{c_{0} D(\kappa)} .
\end{aligned}
$$

This subtraction is compensated by adding the corresponding Sommerfeld integral to the integral over $R_{i k}$ :

$$
\begin{aligned}
G_{i k}\left(\vec{r}, \vec{r}^{\prime}\right) & =\frac{1}{2 \pi} \sum_{n} \frac{c_{i k}^{(n)}}{c_{0}} \frac{1}{\sqrt{P^{2}+z_{i k}^{(n) 2}}}+\frac{1}{2 \pi c_{0}} \int_{0}^{\infty} d \kappa J_{0}(\kappa P) \frac{R_{i k}}{D(\kappa)}, \\
& =\frac{1}{2 \pi} F_{i k}+\ldots \\
G_{i i}\left(\vec{r}, \vec{r}^{\prime}\right) & =\frac{1}{4 \pi \varepsilon_{i}} \sum_{n} \frac{c_{i i}^{(n)}}{c_{0}} \frac{1}{\sqrt{P^{2}+z_{i k}^{(n) 2}}}+\frac{1}{4 \pi \varepsilon_{i} c_{0}} \int_{0}^{\infty} d \kappa J_{0}(\kappa P) \frac{R_{i i}}{D(\kappa)}, \\
i=k & =\frac{1}{4 \pi \varepsilon_{i}} F_{i i}
\end{aligned}
$$

The sum over $n$ in the last but one line, so $F_{i i}$ in the last line, includes also the first term of eq.(62).

Zero values of $z_{i k}^{(n)}$ occur only in the interfaces of the layers and at the boundaries for the offdiagonal pieces $G_{i k}$. For the diagonal pieces these factors in the exponents may be zero across the whole interval to which this piece belongs. The pieces $G_{13}$ and $G_{31}$ are not concerned; in these cases the layer containing the source is separated by layer 2 (= gap) from the layer containing the field point. So the smallest $z_{13}=g$ and $z_{31}=g$ and no cure appears to be necessary.

The critical exponents are found by listing all the exponentials of the numerator of each $g_{i k}$ and inserting there the substitutions $z^{\prime} \rightarrow z$ and then $z \rightarrow 0, z \rightarrow g, z \rightarrow p$ or $z \rightarrow-q$ as appropriate for the boundaries of the layer the piece $G_{i k}$ belongs to. All the critical exponents and the coefficients of the corresponding exponentials are listed in the table below. 


\begin{tabular}{|c|c|c|c|c|}
\hline$G_{i k}$ & $z_{i k}^{(1)}$ & $c_{i k}^{(1)}$ & $z_{i k}^{(2)}$ & $c_{i k}^{(2)}$ \\
\hline \hline$G_{11}$ & $z+z^{\prime}$ & $\left(\varepsilon_{1}-\varepsilon_{2}\right)\left(\varepsilon_{2}+\varepsilon_{3}\right)$ & $2 q+z+z^{\prime}$ & $-\left(\varepsilon_{1}+\varepsilon_{2}\right)\left(\varepsilon_{2}+\varepsilon_{3}\right)$ \\
\hline$G_{21}$ & $z-z^{\prime}$ & $\left(\varepsilon_{2}+\varepsilon_{3}\right)$ & & \\
\hline$G_{31}$ & & & & \\
\hline$G_{12}$ & $z^{\prime}-z$ & $\left(\varepsilon_{2}+\varepsilon_{3}\right)$ & & \\
\hline$G_{22}$ & $z+z^{\prime}$ & $\left(\varepsilon_{1}+\varepsilon_{2}\right)\left(\varepsilon_{2}-\varepsilon_{3}\right)$ & $2 g-z-z^{\prime}$ & $-\left(\varepsilon_{1}-\varepsilon_{2}\right)\left(\varepsilon_{2}+\varepsilon_{3}\right)$ \\
\hline$G_{32}$ & $z-z^{\prime}$ & $\left(\varepsilon_{1}+\varepsilon_{2}\right)$ & & \\
\hline$G_{13}$ & & & & \\
\hline$G_{23}$ & $z^{\prime}-z$ & $\left(\varepsilon_{1}+\varepsilon_{2}\right)$ & & \\
\hline$G_{33}$ & $z+z^{\prime}-2 g$ & $-\left(\varepsilon_{1}+\varepsilon_{2}\right)\left(\varepsilon_{2}-\varepsilon_{3}\right)$ & $2 p-z-z^{\prime}$ & $-\left(\varepsilon_{1}+\varepsilon_{2}\right)\left(\varepsilon_{2}+\varepsilon_{3}\right)$ \\
\hline
\end{tabular}

If one of the layers is very thin (say its thickness is $\delta$ ) it may be commendable or even necessary to subtract exponentials of typ $e^{-\kappa \delta}$. 


\subsection{Final Formulas for the Potential of a Point Charge}

At present only a single point charge $Q$ located at the point $\vec{r}=\vec{r}^{\prime \prime}$ is considered. This single source point may be located in any of the three layers. So the potential, too, must be adorned with two subscripts, the first (second) one indicating the layer where the field (source) point is located. So we have again three cases. In each case one of the electric charge distributions equals $Q \delta\left(\vec{r}^{\prime}-\vec{r}^{\prime \prime}\right)$ while there are no charges in the other two layers. For example, for $Q$ located in the gap we have $\rho_{e 1}=\rho_{e 3}=0, \rho_{e 2}(\vec{r})=Q \delta\left(\vec{r}^{\prime}-\vec{r}^{\prime \prime}\right)$. These different sets of charge distributions are inserted into Eq.(49). There the integrations w.r.t. $\vec{r}^{\prime}$ are done and this variable disappears. Thereafter the notation of the source point $\vec{r}^{\prime \prime}$ may be changed to $\vec{r}^{\prime}$ to give:

$$
\Phi_{i k}(\rho, \phi, z)=Q G_{i k}\left(\rho, \phi, z ; \rho^{\prime}, \phi^{\prime}, z^{\prime}\right) .
$$

While in (1) the dielectric constant was displayed explicitly it is contained in the Green's function in the above expression. The cured integral representations (65) and (66) are used to find numerical values. With $P$ defined as before by

$$
P^{2}=\rho^{2}+\rho^{\prime 2}-2 \rho \rho^{\prime} \cos \left(\phi-\phi^{\prime}\right)
$$

we get the following expressions for the various potentials (i.e. the potential at $\rho, \phi, z$ for a points charge sitting at $\rho^{\prime}, \phi^{\prime}, z^{\prime}$, the first index gives the layer where the potential is evaluated, the second index gives the layer where the charge is sitting):

$$
\begin{aligned}
& \Phi_{11}(\rho, \phi, z)=\frac{Q}{2 \pi \varepsilon_{1}}\left[\frac{1}{2 \sqrt{P^{2}+\left(z-z^{\prime}\right)^{2}}}+\frac{\left(\varepsilon_{1}-\varepsilon_{2}\right)}{2\left(\varepsilon_{1}+\varepsilon_{2}\right) \sqrt{P^{2}+\left(z+z^{\prime}\right)^{2}}}\right. \\
& -\frac{1}{2 \sqrt{P^{2}+\left(2 q+z+z^{\prime}\right)^{2}}} \\
& \left.+\frac{1}{2} \int_{0}^{\infty} d \kappa J_{0}(\kappa P) \frac{R_{11}\left(\kappa, z, z^{\prime}\right)}{\left(\varepsilon_{1}+\varepsilon_{2}\right) D(\kappa)}\right], \quad-q \leq z \leq 0 \\
& \Phi_{21}(\rho, \phi, z)=\frac{Q}{2 \pi} \frac{1}{\left(\varepsilon_{1}+\varepsilon_{2}\right)}\left[\frac{1}{\sqrt{P^{2}+\left(z-z^{\prime}\right)^{2}}}\right. \\
& \left.+\int_{0}^{\infty} d \kappa J_{0}(\kappa P) \frac{R_{21}\left(\tau, z, z^{\prime}\right)}{D(\kappa)}\right], \quad 0 \leq z \leq g \\
& \Phi_{31}(\rho, \phi, z)=\frac{Q}{2 \pi} \int_{0}^{\infty} d \kappa J_{0}(\kappa P) \frac{N_{31}\left(\tau, z, z^{\prime}\right)}{D(\kappa)}, \quad g \leq z \leq p . \\
& \Phi_{12}(\rho, \phi, z)=\frac{Q}{2 \pi} \frac{1}{\left(\varepsilon_{1}+\varepsilon_{2}\right)}\left[\frac{1}{\sqrt{P^{2}+\left(z-z^{\prime}\right)^{2}}}\right. \\
& \begin{array}{r}
\left.+\int_{0}^{\infty} d \kappa J_{0}(\kappa P) \frac{R_{12}\left(\tau, z, z^{\prime}\right)}{D(\kappa)}\right], \quad-q \leq z \leq \\
\Phi_{22}(\rho, \phi, z)=\frac{Q}{2 \pi \varepsilon_{2}}\left[\frac{1}{2 \sqrt{P^{2}+\left(z-z^{\prime}\right)^{2}}}+\frac{\left(\varepsilon_{2}-\varepsilon_{3}\right)}{2\left(\varepsilon_{2}+\varepsilon_{3}\right) \sqrt{P^{2}+\left(2 g-z-z^{\prime}\right)^{2}}}\right.
\end{array} \\
& -\frac{\left(\varepsilon_{1}-\varepsilon_{2}\right)}{2\left(\varepsilon_{1}+\varepsilon_{2}\right) \sqrt{P^{2}+\left(z+z^{\prime}\right)^{2}}}
\end{aligned}
$$




$$
\begin{aligned}
& \left.+\frac{1}{2\left(\varepsilon_{1}+\varepsilon_{2}\right)\left(\varepsilon_{2}+\varepsilon_{3}\right)} \int_{0}^{\infty} d \kappa J_{0}(\kappa P) \frac{R_{22}\left(\tau, z, z^{\prime}\right)}{D(\kappa)}\right], 0 \leq z \leq g ;(73) \\
& \Phi_{32}(\rho, \phi, z)=\frac{Q}{2 \pi} \frac{1}{\left(\varepsilon_{2}+\varepsilon_{3}\right)}\left[\frac{1}{\sqrt{P^{2}+\left(z-z^{\prime}\right)^{2}}}\right. \\
& \left.+\int_{0}^{\infty} d \kappa J_{0}(\kappa P) \frac{R_{32}\left(\tau, z, z^{\prime}\right)}{D(\kappa)}\right], \quad g \leq z \leq p \\
& \Phi_{13}(\rho, \phi, z)=\frac{Q}{2 \pi} \int_{0}^{\infty} d \kappa J_{0}(\kappa P) \frac{N_{13}\left(\kappa, z, z^{\prime}\right)}{D(\kappa)}, \quad-q \leq z \leq 0 \\
& \Phi_{23}(\rho, \phi, z)=\frac{Q}{2 \pi} \frac{1}{\left(\varepsilon_{2}+\varepsilon_{3}\right)}\left[\frac{1}{\sqrt{P^{2}+\left(z-z^{\prime}\right)^{2}}}\right. \\
& \left.+\int_{0}^{\infty} d \kappa J_{0}(\kappa P) \frac{R_{23}\left(\kappa, z, z^{\prime}\right)}{D(\kappa)}\right], \quad 0 \leq z \leq g \\
& \Phi_{33}(\rho, \phi, z)=\frac{Q}{2 \pi \varepsilon_{3}}\left[\frac{1}{2 \sqrt{P^{2}+\left(z-z^{\prime}\right)^{2}}}-\frac{\left(\varepsilon_{2}-\varepsilon_{3}\right)}{2\left(\varepsilon_{2}+\varepsilon_{3}\right) \sqrt{P^{2}+\left(2 g-z-z^{\prime}\right)^{2}}}\right. \\
& -\frac{1}{2 \sqrt{P^{2}+\left(2 p-z-z^{\prime}\right)^{2}}} \\
& \left.+\frac{1}{2} \int_{0}^{\infty} d \kappa J_{0}(\kappa P) \frac{R_{33}\left(\kappa, z, z^{\prime}\right)}{\left(\varepsilon_{2}+\varepsilon_{3}\right) D(\kappa)}\right], \quad g \leq z \leq p .
\end{aligned}
$$

In Eqs.(69) to (77) all terms except for the first one comprising the charge $Q$ have been written such that they are homogeneous in the dielectric constants $\varepsilon_{i}$; so there it does not matter whether one uses the absolute or relative values of these constants.

The denominator of each integrand contains the same function of $\kappa$.

$$
\begin{aligned}
D(\kappa)= & \left(\varepsilon_{1}+\varepsilon_{2}\right)\left(\varepsilon_{2}+\varepsilon_{3}\right)\left(1-e^{-2 \kappa(p+q)}\right) \\
- & \left(\varepsilon_{1}-\varepsilon_{2}\right)\left(\varepsilon_{2}+\varepsilon_{3}\right)\left(e^{-2 \kappa p}-e^{-2 \kappa q}\right) \\
- & \left(\varepsilon_{1}+\varepsilon_{2}\right)\left(\varepsilon_{2}-\varepsilon_{3}\right)\left(e^{-2 \kappa(p-g)}-e^{-2 \kappa(q+g)}\right) \\
+ & \left(\varepsilon_{1}-\varepsilon_{2}\right)\left(\varepsilon_{2}-\varepsilon_{3}\right)\left(e^{-2 \kappa g}-e^{-2 \kappa(p+q-g)}\right) .
\end{aligned}
$$

The first two cured numerators are:

$$
\begin{aligned}
& R_{11}\left(\kappa, z, z^{\prime}\right)= \\
& \left(\varepsilon_{1}-\varepsilon_{2}\right)^{2}\left(\varepsilon_{2}-\varepsilon_{3}\right) e^{\kappa\left(2 g-2 p-2 q+z+z^{\prime}\right)}-\left(\varepsilon_{1}-\varepsilon_{2}\right)^{2}\left(\varepsilon_{2}+\varepsilon_{3}\right) e^{\kappa\left(-2 q+z+z^{\prime}\right)}+ \\
& \left(\varepsilon_{1}+\varepsilon_{2}\right)^{2}\left(\varepsilon_{2}-\varepsilon_{3}\right)\left[e^{\kappa\left(-2 g-4 q-z-z^{\prime}\right)}-e^{\kappa\left(-2 g-2 q+z-z^{\prime}\right)}-e^{\kappa\left(-2 g-2 q-z+z^{\prime}\right)}\right]+ \\
& \left(\varepsilon_{1}+\varepsilon_{2}\right)^{2}\left(\varepsilon_{2}+\varepsilon_{3}\right)\left[-e^{\kappa\left(-2 p-4 q-z-z^{\prime}\right)}+e^{\kappa\left(-2 p-2 q+z-z^{\prime}\right)}+e^{\kappa\left(-2 p-2 q-z+z^{\prime}\right)}\right]+ \\
& 4 \varepsilon_{1} \varepsilon_{2}\left(\varepsilon_{2}-\varepsilon_{3}\right) e^{\kappa\left(-2 g+z+z^{\prime}\right)}-4 \varepsilon_{1} \varepsilon_{2}\left(\varepsilon_{2}+\varepsilon_{3}\right) e^{\kappa\left(-2 p+z+z^{\prime}\right)}+ \\
& \left(\varepsilon_{1}^{2}-\varepsilon_{2}^{2}\right)\left(\varepsilon_{2}-\varepsilon_{3}\right)\left[-e^{\kappa\left(2 g-2 p-4 q-z-z^{\prime}\right)}+e^{\kappa\left(2 g-2 p-2 q+z-z^{\prime}\right)}+e^{\kappa\left(2 g-2 p-2 q-z+z^{\prime}\right)}\right. \\
& \left.-e^{\kappa\left(-2 g-2 q+z+z^{\prime}\right)}\right]+
\end{aligned}
$$




$$
\begin{aligned}
& \left(\varepsilon_{1}^{2}-\varepsilon_{2}^{2}\right)\left(\varepsilon_{2}+\varepsilon_{3}\right)\left[e^{\kappa\left(-4 q-z-z^{\prime}\right)}-e^{\kappa\left(-2 q+z-z^{\prime}\right)}-e^{\kappa\left(-2 q-z+z^{\prime}\right)}+e^{\kappa\left(-2 p-2 q+z+z^{\prime}\right)}\right] \\
& R_{21}\left(\kappa, z, z^{\prime}\right)= \\
& \left(\varepsilon_{1}-\varepsilon_{2}\right)\left(\varepsilon_{2}-\varepsilon_{3}\right)\left[-e^{\kappa\left(-2 g-z+z^{\prime}\right)}+e^{\kappa\left(2 g-2 p-2 q-z+z^{\prime}\right)}\right]+ \\
& \left(\varepsilon_{1}+\varepsilon_{2}\right)\left(\varepsilon_{2}-\varepsilon_{3}\right)\left[e^{\kappa\left(2 g-2 p-2 q-z-z^{\prime}\right)}-e^{\kappa\left(-2 g-2 q+z-z^{\prime}\right)}-e^{\kappa\left(-2 g-2 q-z+z^{\prime}\right)}+e^{\kappa\left(-2 g+z+z^{\prime}\right)}\right]+ \\
& \left(\varepsilon_{1}-\varepsilon_{2}\right)\left(\varepsilon_{2}+\varepsilon_{3}\right)\left[e^{\kappa\left(-2 p-z+z^{\prime}\right)}-e^{\kappa\left(-2 q-z+z^{\prime}\right)}\right]+ \\
& \left(\varepsilon_{1}+\varepsilon_{2}\right)\left(\varepsilon_{2}+\varepsilon_{3}\right)\left[-e^{\kappa\left(-2 q-z-z^{\prime}\right)}+e^{\kappa\left(-2 p-2 q+z-z^{\prime}\right)}+e^{\kappa\left(-2 p-2 q-z+z^{\prime}\right)}-e^{\kappa\left(-2 p+z+z^{\prime}\right)}\right] ;
\end{aligned}
$$

The following numerator needs no cure.

$$
\begin{aligned}
& N_{31}\left(\kappa, z, z^{\prime}\right)= \\
& 2 \varepsilon_{2}\left[e^{-\kappa\left(z-z^{\prime}\right)}+e^{-\kappa\left(2 p+2 q-z+z^{\prime}\right)}-e^{-\kappa\left(2 p-z-z^{\prime}\right)}-e^{-\kappa\left(2 q+z+z^{\prime}\right)}\right] .
\end{aligned}
$$

The next cured numerators are:

$$
\begin{aligned}
& R_{12}\left(\kappa, z, z^{\prime}\right)= \\
& \left(\varepsilon_{1}-\varepsilon_{2}\right)\left(\varepsilon_{2}-\varepsilon_{3}\right)\left[-e^{\kappa\left(-2 g+z-z^{\prime}\right)}+e^{\kappa\left(2 g-2 p-2 q+z-z^{\prime}\right)}\right]+ \\
& \left(\varepsilon_{1}+\varepsilon_{2}\right)\left(\varepsilon_{2}-\varepsilon_{3}\right)\left[e^{\kappa\left(2 g-2 p-2 q-z-z^{\prime}\right)}-e^{\kappa\left(-2 g-2 q+z-z^{\prime}\right)}-e^{\kappa\left(-2 g-2 q-z+z^{\prime}\right)}+e^{\kappa\left(-2 g+z+z^{\prime}\right)}\right]+ \\
& \left(\varepsilon_{1}-\varepsilon_{2}\right)\left(\varepsilon_{2}+\varepsilon_{3}\right)\left[e^{\kappa\left(-2 p+z-z^{\prime}\right)}-e^{\kappa\left(-2 q+z-z^{\prime}\right)}\right]+ \\
& \left(\varepsilon_{1}+\varepsilon_{2}\right)\left(\varepsilon_{2}+\varepsilon_{3}\right)\left[-e^{\kappa\left(-2 q-z-z^{\prime}\right)}+e^{\kappa\left(-2 p-2 q+z-z^{\prime}\right)}+e^{\kappa\left(-2 p-2 q-z+z^{\prime}\right)}-e^{\kappa\left(-2 p+z+z^{\prime}\right)}\right] ; \\
& R_{22}\left(\kappa ; z, z^{\prime}\right)= \\
& \left(\varepsilon_{1}+\varepsilon_{2}\right)^{2}\left(\varepsilon_{2}+\varepsilon_{3}\right)^{2}\left[e^{\kappa\left(-2 p-2 q+z-z^{\prime}\right)}+e^{\kappa\left(-2 p-2 q-z+z^{\prime}\right)}\right]- \\
& \left(\varepsilon_{1}+\varepsilon_{2}\right)^{2}\left(\varepsilon_{2}-\varepsilon_{3}\right)^{2} e^{\kappa\left(-4 g-2 q+z+z^{\prime}\right)}- \\
& 4 \varepsilon_{1} \varepsilon_{2}\left(\varepsilon_{2}+\varepsilon_{3}\right)^{2} e^{\kappa\left(-2 q-z-z^{\prime}\right)}-\left(\varepsilon_{1}-\varepsilon_{2}\right)^{2}\left(\varepsilon_{2}+\varepsilon_{3}\right)^{2} e^{\kappa\left(-2 p-z-z^{\prime}\right)}- \\
& \left(\varepsilon_{1}^{2}-\varepsilon_{2}^{2}\right)\left(\varepsilon_{2}-\varepsilon_{3}\right)^{2} e^{\kappa\left(-4 g+z+z^{\prime}\right)}+ \\
& \left(\varepsilon_{1}{ }^{2}-\varepsilon_{2}{ }^{2}\right)\left(\varepsilon_{2}+\varepsilon_{3}\right)^{2}\left[-e^{\kappa\left(-2 p-2 q-z-z^{\prime}\right)}+e^{\kappa\left(-2 p+z-z^{\prime}\right)}+e^{\kappa\left(-2 p-z+z^{\prime}\right)}\right]- \\
& 4\left(\varepsilon_{1}{ }^{2}-\varepsilon_{2}{ }^{2}\right) \varepsilon_{2} \varepsilon_{3} e^{\kappa\left(-2 p-2 q+z+z^{\prime}\right)}-4\left(\varepsilon_{1}+\varepsilon_{2}\right)^{2} \varepsilon_{2} \varepsilon_{3} e^{\kappa\left(-2 p+z+z^{\prime}\right)}+ \\
& \left(\varepsilon_{1}-\varepsilon_{2}\right)^{2}\left(\varepsilon_{2}{ }^{2}-\varepsilon_{3}{ }^{2}\right) e^{\kappa\left(-2 g-z-z^{\prime}\right)}+4 \varepsilon_{1} \varepsilon_{2}\left(\varepsilon_{2}{ }^{2}-\varepsilon_{3}{ }^{2}\right) e^{\kappa\left(2 g-2 p-2 q-z-z^{\prime}\right)}+ \\
& \left(\varepsilon_{1}+\varepsilon_{2}\right)^{2}\left(\varepsilon_{2}{ }^{2}-\varepsilon_{3}{ }^{2}\right)\left[-e^{\kappa\left(-2 g-2 q+z-z^{\prime}\right)}-e^{\kappa\left(-2 g-2 q-z+z^{\prime}\right)}+e^{\kappa\left(-2 g-2 p-2 q+z+z^{\prime}\right)}\right]+ \\
& \left(\varepsilon_{1}{ }^{2}-\varepsilon_{2}{ }^{2}\right)\left(\varepsilon_{2}{ }^{2}-\varepsilon_{3}{ }^{2}\right)\left[e^{\kappa\left(-2 g-2 q-z-z^{\prime}\right)}-e^{\kappa\left(-2 g+z-z^{\prime}\right)}-e^{\kappa\left(-2 g-z+z^{\prime}\right)}+e^{\kappa\left(-2 g-2 p+z+z^{\prime}\right)}\right] ; \\
& R_{32}\left(\kappa, z, z^{\prime}\right)=
\end{aligned}
$$




$$
\begin{aligned}
& \left(\varepsilon_{1}-\varepsilon_{2}\right)\left(\varepsilon_{2}-\varepsilon_{3}\right)\left[-e^{\kappa\left(-2 g-z+z^{\prime}\right)}+e^{\kappa\left(2 g-2 p-2 q-z+z^{\prime}\right)}\right]+ \\
& \left(\varepsilon_{1}+\varepsilon_{2}\right)\left(\varepsilon_{2}-\varepsilon_{3}\right)\left[e^{\kappa\left(2 g-2 p-z+z^{\prime}\right)}-e^{\kappa\left(-2 g-2 q-z+z^{\prime}\right)}\right]+ \\
& \left(\varepsilon_{1}-\varepsilon_{2}\right)\left(\varepsilon_{2}+\varepsilon_{3}\right)\left[-e^{\kappa\left(-z-z^{\prime}\right)}+e^{\kappa\left(-2 p+z-z^{\prime}\right)}+e^{\kappa\left(-2 p-z+z^{\prime}\right)}-e^{\kappa\left(-2 p-2 q+z+z^{\prime}\right)}\right]+ \\
& \left(\varepsilon_{1}+\varepsilon_{2}\right)\left(\varepsilon_{2}+\varepsilon_{3}\right)\left[-e^{\kappa\left(-2 q-z-z^{\prime}\right)}+e^{\kappa\left(-2 p-2 q+z-z^{\prime}\right)}+e^{\kappa\left(-2 p-2 q-z+z^{\prime}\right)}-e^{\kappa\left(-2 p+z+z^{\prime}\right)}\right]
\end{aligned}
$$

The following numerator needs no cure:

$$
\begin{aligned}
& N_{13}\left(\kappa, z, z^{\prime}\right)= \\
& 2 \varepsilon_{2}\left(e^{\kappa\left(z-z^{\prime}\right)}+e^{-\kappa\left(2 p+2 q+z-z^{\prime}\right)}-e^{-\kappa\left(2 p-z-z^{\prime}\right)}-e^{-\kappa\left(2 q+z+z^{\prime}\right)}\right) .
\end{aligned}
$$

The last cured numerators are:

$$
\begin{aligned}
& R_{23}\left(\kappa, z, z^{\prime}\right)= \\
& \left(\varepsilon_{1}-\varepsilon_{2}\right)\left(\varepsilon_{2}-\varepsilon_{3}\right)\left[-e^{\kappa\left(-2 g+z-z^{\prime}\right)}+e^{\kappa\left(2 g-2 p-2 q+z-z^{\prime}\right)}\right]+ \\
& \left(\varepsilon_{1}+\varepsilon_{2}\right)\left(\varepsilon_{2}-\varepsilon_{3}\right)\left[e^{\kappa\left(2 g-2 p+z-z^{\prime}\right)}-e^{\kappa\left(-2 g-2 q+z-z^{\prime}\right)}\right]+ \\
& \left(\varepsilon_{1}-\varepsilon_{2}\right)\left(\varepsilon_{2}+\varepsilon_{3}\right)\left[-e^{\kappa\left(-z-z^{\prime}\right)}+e^{\kappa\left(-2 p+z-z^{\prime}\right)}+e^{\kappa\left(-2 p-z+z^{\prime}\right)}-e^{\kappa\left(-2 p-2 q+z+z^{\prime}\right)}\right]+ \\
& \left(\varepsilon_{1}+\varepsilon_{2}\right)\left(\varepsilon_{2}+\varepsilon_{3}\right)\left[-e^{\kappa\left(-2 q-z-z^{\prime}\right)}+e^{\kappa\left(-2 p-2 q+z-z^{\prime}\right)}+e^{\kappa\left(-2 p-2 q-z+z^{\prime}\right)}-e^{\kappa\left(-2 p+z+z^{\prime}\right)}\right] \\
& R_{33}\left(\kappa, z, z^{\prime}\right)= \\
& -4\left(\varepsilon_{1}+\varepsilon_{2}\right) \varepsilon_{2} \varepsilon_{3} e^{\kappa\left(-2 q-z-z^{\prime}\right)}-4\left(\varepsilon_{1}-\varepsilon_{2}\right) \varepsilon_{2} \varepsilon_{3} e^{\kappa\left(-z-z^{\prime}\right)}- \\
& \left(\varepsilon_{1}-\varepsilon_{2}\right)\left(\varepsilon_{2}-\varepsilon_{3}\right)^{2} e^{\kappa\left(4 g-2 p-2 q-z-z^{\prime}\right)}-\left(\varepsilon_{1}+\varepsilon_{2}\right)\left(\varepsilon_{2}-\varepsilon_{3}\right)^{2} e^{\kappa\left(4 g-2 p-z-z^{\prime}\right)}+ \\
& \left(\varepsilon_{1}-\varepsilon_{2}\right)\left(\varepsilon_{2}+\varepsilon_{3}\right)^{2}\left[e^{\kappa\left(-2 p+z-z^{\prime}\right)}+e^{\kappa\left(-2 p-z+z^{\prime}\right)}-e^{\kappa\left(-4 p+z+z^{\prime}\right)}\right]+ \\
& \left(\varepsilon_{1}+\varepsilon_{2}\right)\left(\varepsilon_{2}+\varepsilon_{3}\right)^{2}\left[e^{\kappa\left(-2 p-2 q+z-z^{\prime}\right)}+e^{\kappa\left(-2 p-2 q-z+z^{\prime}\right)}-e^{\kappa\left(-4 p-2 q+z+z^{\prime}\right)}\right]+ \\
& \left(\varepsilon_{1}-\varepsilon_{2}\right)\left(\varepsilon_{2}^{2}-\varepsilon_{3}^{2}\right)\left[-e^{\kappa\left(2 g-2 p-z-z^{\prime}\right)}+e^{\kappa\left(2 g-2 p-2 q+z-z^{\prime}\right)}+\right. \\
& \left(\varepsilon_{1}+\varepsilon_{2}\right)\left(\varepsilon_{2}^{2}-\varepsilon_{3}^{2}\right)\left[-e^{\kappa\left(2 g-2 p-2 q-z-z^{\prime}\right)}+e^{\kappa\left(2 g-2 p+z-z^{\prime}\right)}+\right. \\
& \left.+e^{\kappa\left(2 g-2 p-2 q-z+z^{\prime}\right)}-e^{\kappa\left(2 g-4 p-2 q+z+z^{\prime}\right)}\right]+ \\
& +\left(2 g-2 p-z+z^{\prime}\right) \\
& \left.+e^{\kappa\left(2 g-4 p+z+z^{\prime}\right)}\right]
\end{aligned}
$$

The cure by subtraction entails that the number of exponentials in the numerators increases substantially.

\subsubsection{Computation of the Electric Field Components}

The expressions for the electric fields are found from the potentials by derivation. For the radial electric field component $E_{\rho}$ the integrals contain $-\kappa J_{1}(\kappa \rho)$ in place of $J_{0}(\kappa \rho)$. Since this $\kappa$ is multiplied by exponentials with negative exponents the convergence may be a bit slower than that of the integrads of the potential but will still be satisfactory. The very last remark applies 
also to the component $E_{z}$. But all the numerators $R_{i k}\left(\kappa, z, z^{\prime}\right)$ (or $N_{i k}\left(\kappa, z, z^{\prime}\right)$ ) must be derived with respect to z. Of course, all the derivations of the terms with square roots in front of the integrals must be done.

\subsubsection{Problems with Series Representations}

In principle also series representations may be derived for the Green's function in a plane condensor comprising several homogeneous layers. This has been done before [10], [16]. But this requires to find a large number of roots of a transcendental equation; and it must be ensured that all roots with small values are found! In our case this is the equation $D(\kappa)=0$ with the function $D$ defined in eq.(78). The resulting series converge slowly or not at all (those for the field components) and must be summed and differentiated numerically. So such an approach has unfavourable auspices.

\subsection{The Weighting Field in the Gas Gap}

As in subsect.2.3 and under the same presumptions (infinitely thin and infinitely long strip, gaps between strips neglected) the weighting field in the gap (layer 2) is computed for a strip electrode $-w / 2 \leq x \leq w / 2,-\infty \leq y \leq \infty, z=-q<0$. The integral representation (54) of the Green's function, Green's generalized theorem, eq.(47), are used together with the generalized Laplace equation (42) for the potential, the differential equation (45) and the homogeneous boundary condition for the Green's function. The potential equals $V_{1}$ along the strip (or patch) and is zero elsewhere. This gives the following result for the weighting potential:

$$
\Phi_{1}(x, z)=\left.V_{1} \varepsilon_{1} \frac{2}{\pi} \int_{0}^{\infty} d \kappa \cos (\kappa x) \sin \left(\kappa \frac{w}{2}\right) \frac{1}{\kappa} \frac{\partial g_{21}\left(\kappa ; z, z^{\prime}\right)}{\partial z^{\prime}}\right|_{z^{\prime}=-q}
$$

The two components of the weighting field are given by:

$$
\begin{aligned}
& E_{x}(x, z)=-\left.V_{1} \varepsilon_{1} \frac{2}{\pi} \int_{0}^{\infty} d \kappa \sin (\kappa x) \sin \left(\kappa \frac{w}{2}\right) \frac{\partial g_{21}\left(\kappa ; z, z^{\prime}\right)}{\partial z^{\prime}}\right|_{z^{\prime}=-q} \\
& E_{z}(x, z)=\left.V_{1} \varepsilon_{1} \frac{2}{\pi} \int_{0}^{\infty} d \kappa \cos (\kappa x) \sin \left(\kappa \frac{w}{2}\right) \frac{1}{\kappa} \frac{\partial^{2} g_{21}\left(\kappa ; z, z^{\prime}\right)}{\partial z \partial z^{\prime}}\right|_{z^{\prime}=-q} .
\end{aligned}
$$

with

$$
\begin{aligned}
& \left.\frac{\partial g_{21}\left(\kappa ; z, z^{\prime}\right)}{\partial z^{\prime}}\right|_{z^{\prime}=-q}= \\
& \frac{2}{D(\kappa)}\left[\left(\varepsilon_{2}+\varepsilon_{3}\right)\left(e^{-\kappa(q+z)}-e^{-\kappa(2 p+q-z)}\right)+\left(\varepsilon_{2}-\varepsilon_{3}\right)\left(e^{-\kappa(2 g+q-z)}-e^{-\kappa(2 p+q-2 g+z)}\right)\right]
\end{aligned}
$$

and

$$
\begin{aligned}
& \left.\frac{1}{\kappa} \frac{\partial^{2} g_{21}\left(\kappa ; z, z^{\prime}\right)}{\partial z^{\prime} \partial z}\right|_{z^{\prime}=-q}= \\
& -\frac{2}{D(\kappa)}\left[\left(\varepsilon_{2}+\varepsilon_{3}\right)\left(e^{-\kappa(q+z)}+e^{-\kappa(2 p+q-z)}\right)-\left(\varepsilon_{2}-\varepsilon_{3}\right)\left(e^{-\kappa(q+2 g-z)}+e^{-\kappa(2 p+q-2 g+z)}\right)\right] .
\end{aligned}
$$

The above integrals must be evaluated numerically. No cure is needed as long as layer 1 is not too thin, i.e. if $q$ is not too small. If the strip is very wide the field $E_{z}$ in the center of the strip $(x=0)$ has to approach

$$
E_{z}=\frac{V_{1} \varepsilon_{1} \varepsilon_{3}}{\varepsilon_{2} \varepsilon_{3} q+\varepsilon_{1} \varepsilon_{2} p+\left(\varepsilon_{1} \varepsilon_{3}-\varepsilon_{1} \varepsilon_{2}\right) g}
$$


independent of $z$, which is derived simply from assuming an infinite plane capacitor. Setting $x=0$, substituting $r=\kappa w / 2$ and performing $w \rightarrow \infty$ in Eq. (91) we actually arrive at Eq. (94).

\section{Summary and Applications}

Analytic expressions for the electric field of a point charge in an infinite plane condensor with one or three homogeneous layers were derived.

The final expressions for the field in the one-layer geometry are given in Equations 29 and 30. Weigthing fields for a strip electrode are given in Equations 36 and 37.

The final expressions for the field in the three-layer geometry are given in Equations 69 to 88 . Weigthing fields for a strip electrode are given in Equations 90 to 93.

The field solutions allow e.g. the calculation of space charge effects within the electron avalanches in Parallel Plate Chambers or Resistive Plate Chambers which are responsible for streamer effects and breakdown [2]. Also the efficiency drop in Resistive Plate Chambers at high counting rates can be calculated with these solutions since this effect is due to the electric field of charges on the surface of the resistive plates.

The weighting field solutions allow the calculation of induced signals and cross-induced signals e.g. for an RPC with fine pitch strips for position measurements using the center of gravity method.

\section{References}

[1] V. Fanti et al. [NA48 Collaboration], "Performance of an electromagnetic liquid krypton calorimeter," Nucl. Instrum. Meth. A 344, 507 (1994).

[2] H. Raether, Electron Avalanches and Breakdown in Gases, London, U.K. Butterworth, 1964

[3] International workshops on Resistive Plate Chambers and related detectors, Coimbra 2001, 1999, 1997, 1995, 1993

[4] S. Wolfram, The Mathematica book, Cambridge Univ.Press, Cambridge, 1996.

[5] M. Abramowitz and I. Stegun, Handbook of Mathematical Functions.

[6] A. Sommerfeld, Vorlesungen über Theoretische Physik, Vol VI: Partielle Differentialgleichungen der Physik. Akademische Verlagsgesellschaft, Leipzig 1958.

[7] G. N. Watson, A Treatise on the Theory of Bessel Functions. Cambridge, University Press, 1966.

[8] F. E. Borgnis, Ch. H. Papas, Randwertprobleme der Mikrowellenphysik. Springer, Berlin, 1955. $\S 2.5$

[9] E. A. Kraut, Fundmentals of Mathematical Physics. McGraw-Hill, New York, 1967.

[10] Th. Heubrandtner, Theoretical Models for Signal Generation in Resistive Plate Chambers. Dissertation, Technisch-Naturwissenschaftliche Fakultät, Technische Universität Graz, 1999.

[11] S. Ramo, Currents induced in electron motion, PROC. IRE 27, 584 (1939)

[12] W. Blum, L. Rolandi: Particle Detection with Drift Chambers. Springer Verlag 1994.

[13] E. Gatti, G. Padovini, V. Radeka, Signal Evaluation in Multielectrode Radiation Detectors by Means of a Time Dependent Weighting Vector. NIMA 193 (1982) 651-3.

[14] I.S. Gradshteyn, I.M. Ryzhik, Table of Integrals, Series, and Products. Academic Press, Nww York and London, 1965. 3.981.5-8; 4.114.1 . 
[15] W. A. Newcomb, Trouble with the method of images. Am. J. Phys. 50 (\# 7, July 1982) 601-607.

[16] H. Schöpf, B. Schnizer, Theory Describing Cathode Signals from Charges Moving in Counters with a Poorly Conducting Cathode, Nucl. Instr. and Meth. A 323 (1992) 338 - 344. 